
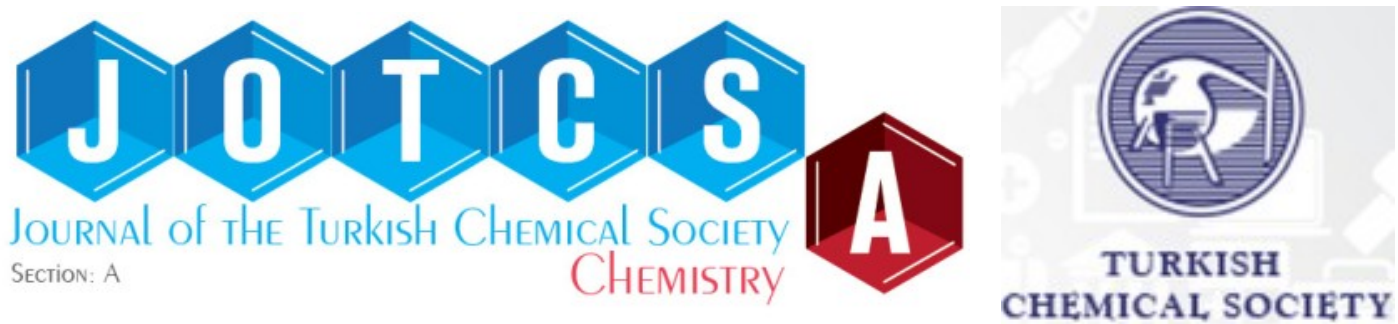

\title{
Dioxomolybdenum(VI) Complexes of ONN-Chelating Thiosemicarbazones: Crystallographic and Spectroscopic (UV, IR and NMR) Studies
}

\author{
Songül Eğlence-Bakıra* \\ aDepartment of Chemistry, Faculty of Science, Istanbul University, 34134, Istanbul, Turkey
}

\begin{abstract}
Four new cis-dioxomolybdenum(VI) complexes of the closed formula $\left[\mathrm{MoO}_{2}(\mathrm{~L}) \mathrm{CH}_{3} \mathrm{OH}\right]$ have been obtained by the condensation of bis(acetylacetonato)dioxomolybdenum(VI) with Sallyl/propyl thiosemicarbazones in methanol. The compounds were identified with elemental analysis, and molecular characterization by UV-Vis, FT-IR, ${ }^{1} \mathrm{H}-\mathrm{NMR}$ and ${ }^{13} \mathrm{C}-\mathrm{NMR}$ spectroscopies. Also, crystal structures of $\mathbf{1}$ and $\mathbf{2}$ were resolved by X-ray diffraction analysis. The single crystal studies indicated a distorted octahedral geometry for complexes. It was noticed that $\mathbf{1}$ crystallized in the monoclinic $\mathrm{P} 2_{1} / \mathrm{c}$ space group with $\mathrm{Z}=4$, while $\mathbf{2}$ crystallized in tricilinic $\mathrm{P}-1$ space group with $\mathrm{Z}=2$. The $\mathrm{O}=\mathrm{Mo}=\mathrm{O}$ angles of $105.68(8)^{\circ}$ (for 1) and $105.96(7)^{\circ}$ (for 2) indicated the cisdioxomolybdenum structure. The spectroscopic and crystallographic studies show that the thiosemicarbazone ligands are bound to $\mathrm{cis}-\mathrm{MoO}_{2}{ }^{2+}$ moiety as ONN tridentate agent and the $6^{\text {th }}$ coordinated position of the molybdenum is located by the methanol molecule.
\end{abstract}

Keywords: Thiosemicarbazone; dioxomolybdenum(VI) complex; X-ray crystallography; spectroscopic studies; structural analysis.

Submitted: September 01, 2021. Accepted: November 13, 2021.

Cite this: Eğlence-Bakır S. Dioxomolybdenum(VI) Complexes of ONN-Chelating Thiosemicarbazones: Crystallographic and Spectroscopic (UV, IR and NMR) Studies. JOTCSA. $2021 ; 8(4): 1275-300$.

DOI: https://doi.org/10.18596/jotcsa.989318.

*Corresponding author. E-mail: songul@istanbul.edu.tr.

\section{INTRODUCTION}

Thiosemicarbazones, obtained through the condensation reaction of thiosemicarbazides with an an aldehyde or a ketone, are one of the main subgroups of hydrazones $(1,2)$. After their activities on living systems was discovered in 1950s, investigators have exhibited an increased attention in thiosemicarbazones and their metal complexes (3). Thiosemicarbazonemetal complexes are an important group of compounds with numerous biological activities (4-13). Thiosemicarbazones can behave as monodentate $(14,15)$, bidentate $(16,17)$, tridentate (18-20), or multidentate $(21,22)$ ligands. Common metal complexes of thiosemicarbazones have tridentate ONS or ONN functions. In both forms, the oxygen atom of an arylidene moiety and the azomethine nitrogen coordinate to the metal ion. Also, the sulfur atom or the thioamide nitrogen coordinate to metal center for ONS- or ONNcoordinated complexes, respectively $(12,18)$.

Molybdenum is a special cofactor for molybdoenzymes such as sulfite oxidase and xanthine oxidase (23). These molybdoenzymes have oxo-groups and the molybdenum ion has 
an oxidation state of (VI) or (IV) during the reaction cycle (24). The solvate dioxomolybdenum(VI) complexes of thiosemicarbazones can behave as catalysts. It is because the solvent molecule may be relocated with the activated enzyme molecule. Several chelates containing cis- $\mathrm{MoO}_{2}{ }^{2+}$ moiety have been obtained for explain the structure of molybdoenzymes and oxygen atom transfer (OAT) reactions (25). As mentioned above, molybdenum complexes are used as models in many biological activity studies, primarily in enzyme systems. In order to study in detail how the activity changes with the structure, it becomes important to systematically synthesize and characterize compounds with different groups on both the aromatic ring, the amide or sulfur atoms and different solvents coordinated to the sixth site of the molybdenum. In early studies, the solvated mononuclear dioxomolybdenum(VI) complexes of thiosemicarbazones containing pyridine (26), acetonitrile (27), methanol (18), ethanol (25), Y-picoline (28), dimethylsulfoxide (29) as solvents have been reported.
The current study was focused on obtain and characterize the ONN-coordinated dioxomolybdenum(VI) complexes of S-allyl/propylthiosemicarbazones. For this purpose, different substitutions have been studied on the aromatic ring, amide nitrogen, and the sulfur atom (Figure 1). The characterization results of ligands and complexes were determined by elemental analysis, UV-Vis, IR, ${ }^{1} \mathrm{H}-\mathrm{NMR}$ and ${ }^{13} \mathrm{C}-\mathrm{NMR}$ spectroscopies.

The structures of cis-dioxo-(S-allyl-N $\mathrm{N}^{1}-2-$ hydroxy-4-methoxybenzylidene-

thiosemicarbazonato ) (O,N, $\left.\mathrm{N}^{\prime}\right)$-methanolmolybdenum(VI) (1) and cis-dioxo-(S-allyl-N ${ }^{1}$ 2-hydroxy-5-chlorobenzylidene- $\mathrm{N}^{4}$-ethyl

thiosemicarbazonato) (O,N,N')-methanolmolybdenum(VI) (2) were also determined by $X$-ray diffraction analysis. Effects of different substitutions on structures were compared using the crystallographic and spectroscopic studies.

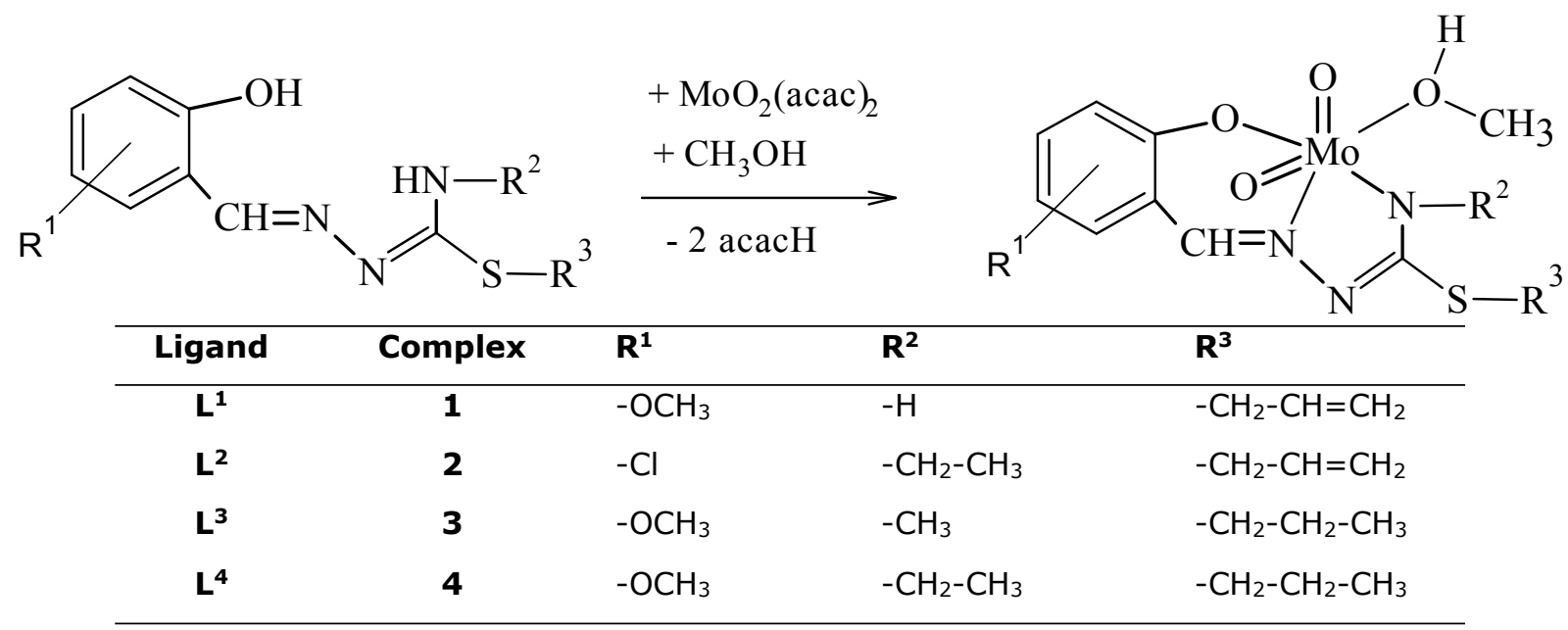

Figure 1: Formation of the complexes.

\section{EXPERIMENTAL}

\section{Materials and Physical Measurements}

Thermo-Finnigan Flash EA 1112 Series Elementary Analyzer was used for record elemental analyses. UV-Vis spectra of the thiosemicarbazones and complexes were performed in chloroform, $3 \times 10^{-5} \mathrm{M}$, with a Shimadzu 2600 UV-Vis spectrophotometer. The infrared spectra of the compounds were obtained on a Bruker Alpha II compact Fourier Transform Infrared (FTIR) spectrometer in range of 4000 to $400 \mathrm{~cm}^{-1}$. Varian VNMRJ 600 Nuclear Magnetic Resonance (NMR) spectrometer was used for the measurements of ${ }^{1} \mathrm{H}-\mathrm{NMR}$ and ${ }^{13} \mathrm{C}-\mathrm{NMR}$ using $\mathrm{CDCl}_{3}$ as solvent. The X-ray intensity data were recorded on a Bruker APEX-II CCD imaging plate area detector with graphite monochromatic Mo-K a radiation $(\lambda=0.71073 \AA)$.

\section{Synthesis of Thiosemicarbazones}

The thiosemicarbazones were obtained for the first time with very minor modifications from known procedures $(18,30)$. The synthesis method was explained as follows by the 2hydroxy-4-methoxybenzylidene-S-

allylthiosemicarbazone $\left(\mathbf{L}^{\mathbf{1}}\right)$ as representative 
example. 0.5 gram (5 millimoles) of thiosemicarbazide and 0.47 milliliter (5 millimoles) of allyl bromide were mixed up in 15 milliliters of ethanol at about $75^{\circ} \mathrm{C}$. The reaction formation was controlled by chromatography. After two hours, the four milliliters of alcoholic solution of 2-hydroxy-4methoxybenzaldehyde ( 0.83 gram, 5 millimol) was added dropwise to colourless mix. An aqueous solution of sodium bicarbonate was added for neutralization of the hydrogen bromide form. The yellow-colored product precipitated from the reaction medium was filtered, washed first with a few drops of ethanol, and then water. The obtained product was recrystallized from ethanol after drying. Other thiosemicarbazones were obtained by using $\mathrm{N}$-methylthiosemicarbazide $\left(\mathbf{L}^{\mathbf{3}}\right), \mathrm{N}$ ethylthiosemicarbazide $\left(\mathbf{L}^{\mathbf{2}}, \mathbf{L}^{\mathbf{4}}\right)$, bromopropane $\left(\mathbf{L}^{\mathbf{3}}, \mathbf{L}^{\mathbf{4}}\right)$ and 5 -chlorosalicylaldehyde $\left(\mathbf{L}^{\mathbf{2}}\right)$ with the same procedure. The colors, melting points $\left({ }^{\circ} \mathrm{C}\right), \%$ yields, elemental analysis, UV-Vis, IR, ${ }^{1} \mathrm{H}-\mathrm{NMR}$ and ${ }^{13} \mathrm{C}-\mathrm{NMR}$ data of the ligands are given below:

L1: Yellow, m.p. 186.2-187.4 ${ }^{\circ} \mathrm{C}$, yield $75 \%$. Anal. Calc. for $\mathrm{C}_{12} \mathrm{H}_{15} \mathrm{~N}_{3} \mathrm{O}_{2} \mathrm{~S}(265.33 \mathrm{~g} / \mathrm{mol})$ : C, 54.32; H, 5.70; N, 15.84; S, 12.08. Found: C, $54.15 ; H, 5.52 ; \mathrm{N}, 15.69 ; \mathrm{S}, 12.23 \%$. UV-Vis $(\mathrm{nm}(\log \varepsilon)): 240 \quad(3.40) ; 295$ (3.36); 305 (3.45); 338 (3.66) (Fig.S1). IR $\left(\mathrm{cm}^{-1}\right): \mathrm{v}(\mathrm{OH})$ 3350; $v_{a s}\left(\mathrm{NH}_{2}\right) 3414 ; \mathrm{v}_{\mathrm{s}}\left(\mathrm{NH}_{2}\right) 3302 ; \mathrm{v}(\mathrm{CH})_{\text {aliphatic }}$ 2960, 2834; v(C-O) 1160; v(C-N) 993; v(C-S) 836; $\mathrm{v}\left(\mathrm{C}=\mathrm{N}^{1}\right)$ 1627; $\mathrm{v}\left(\mathrm{C}=\mathrm{N}^{2}\right) 1602$ (Fig.S5). ${ }^{1} \mathrm{H}-$ NMR (ppm): 11.75, $11.59(\mathrm{~s}, \mathrm{i}: 1 / 1,1 \mathrm{H},-\mathrm{OH})$; 8.37, $8.23\left(\mathrm{~s}, \mathrm{i}: 2 / 1,1 \mathrm{H}, \mathrm{CH}=\mathrm{N}^{1}\right) ; 7.13(\mathrm{~m}, 1 \mathrm{H}$, d); $6.47(\mathrm{~m}, 2 \mathrm{H}, \mathrm{a}, \mathrm{c}) ; 5.08\left(\mathrm{~s}, 2 \mathrm{H}, \mathrm{N}^{4} \mathrm{H}_{2}\right) ; 3.79$ $\left(\mathrm{s}, 3 \mathrm{H},-\mathrm{OCH}_{3}\right) ; 3.71,3.60\left(\mathrm{~d}, 2 \mathrm{H}, \mathrm{S}-\mathrm{C}^{1} \mathrm{H}_{2}-\right)$; $5.98\left(\mathrm{~m}, 1 \mathrm{H},-\mathrm{C}^{2} \mathrm{H}=\right) ; 5.29,5.15\left(\mathrm{~d}, 2 \mathrm{H},=\mathrm{C}^{3} \mathrm{H}_{2}\right)$ (Fig.S13). ${ }^{13} \mathrm{C}-\mathrm{NMR}$ (ppm): 165.35 (=CN-S); $163.40(-\mathrm{CH}=\mathrm{N}), 160.76,158.05,135.35$, $114.66,109.46,103.75$ (aromatics); 58.20 ($\left.\mathrm{OCH}_{3}\right) ; 36.01\left(\mathrm{~S}-\mathrm{CH}_{2}-\right) ; 136.21(-\mathrm{CH}=) ; 120.95$ $\left(=\mathrm{CH}_{2}\right)($ Fig.S21).

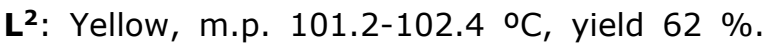
Anal. Calc. for $\mathrm{C}_{13} \mathrm{H}_{16} \mathrm{CIN}_{3} \mathrm{OS}(297.80 \mathrm{~g} / \mathrm{mol})$ : C, 52.43; $\mathrm{H}, 5.42 ; \mathrm{N}, 14.11 ; \mathrm{S}, 10.77$. Found: $\mathrm{C}$, $52.25 ; \mathrm{H}, 5.52 ; \mathrm{N}, 14.26 ; \mathrm{S}, 10.63 \%$. UV-Vis $(\mathrm{nm}(\log \varepsilon)): 240 \quad(3.83) ; 294 \quad(3.80) ; 305$ (3.89); 336 (4.10) (Fig.S2). IR $\left(\mathrm{cm}^{-1}\right): \mathrm{v}(\mathrm{OH})$ 3407; $\mathrm{v}(\mathrm{NH})$ 3394; $\mathrm{v}(\mathrm{CH})$ aliphatic 2970, 2885; $\mathrm{v}(\mathrm{C}-\mathrm{O})$ 1186; $\mathrm{v}(\mathrm{C}-\mathrm{N})$ 935; $\mathrm{v}(\mathrm{C}-\mathrm{S})$ 861; $\mathrm{v}\left(\mathrm{C}=\mathrm{N}^{1}\right)$ 1629; $\mathrm{v}\left(\mathrm{C}=\mathrm{N}^{2}\right) 1600$ (Fig.S7). ${ }^{1} \mathrm{H}-\mathrm{NMR}$ (ppm): $11.77,11.41(\mathrm{~s}, \mathrm{i}: 3 / 1,1 \mathrm{H},-\mathrm{OH}) ; 8.31,8.29$ (s, $\left.\mathrm{i}: 1 / 3,1 \mathrm{H}, \mathrm{CH}=\mathrm{N}^{1}\right) ; 7.17(\mathrm{~m}, 2 \mathrm{H}, b, d) ; 6.90$ $(\mathrm{m}, 2 \mathrm{H}, \mathrm{a}) ; 5.45,4.67\left(\mathrm{t}, \mathrm{i}: 1 / 2,1 \mathrm{H}, \mathrm{N}^{4} \mathrm{H}\right) ; 3.42$, $3.32\left(\mathrm{~m}, \mathrm{i}: 3 / 1,2 \mathrm{H}, \mathrm{N}^{4}-\mathrm{CH}_{2}\right) ; 1.21\left(\mathrm{t}, 3 \mathrm{H},-\mathrm{CH}_{3}\right)$; $3.76,3.54\left(\mathrm{~d}, 2 \mathrm{H}, \mathrm{S}-\mathrm{C}^{1} \mathrm{H}_{2}\right)$; $5.95(\mathrm{~m}, 1 \mathrm{H}$, -
$\left.\mathrm{C}^{2} \mathrm{H}=\right)$; 5.36, 5.27 (d, 2H, $\left.=\mathrm{C}^{3} \mathrm{H}_{2}\right)$ (Fig.S15). ${ }^{13} \mathrm{C}-\mathrm{NMR}(\mathrm{ppm})$ : 164.26 (=CN-S); 159.87 ($\mathrm{CH}=\mathrm{N}), \quad 156.04,132.91,132.95,126.24$, $122.74,121.56$ (aromatics); $36.41\left(\mathrm{~S}-\mathrm{CH}_{2}^{-}\right)$; $136.75(-\mathrm{CH}=) ; 120.57 \quad\left(=\mathrm{CH}_{2}\right) ; 41.26$ (N$\mathrm{CH}_{2}-$ ); $17.04\left(-\mathrm{CH}_{3}\right)$ (Fig.S23).

L3: Yellow, m.p. 185.5-186.2 ${ }^{\circ} \mathrm{C}$, yield $87 \%$. Anal. Calc. for $\mathrm{C}_{13} \mathrm{H}_{19} \mathrm{~N}_{3} \mathrm{O}_{2} \mathrm{~S}(281.37 \mathrm{~g} / \mathrm{mol})$ : C, 55.49; H, 6.81; N, 14.93; S, 11.40. Found: C, $55.25 ; \mathrm{H}, 6.72 ; \mathrm{N}, 14.79 ; \mathrm{S}, 11.23 \%$. UV-Vis $(\mathrm{nm} \quad(\log \varepsilon)): 240 \quad(4.03) ; 294 \quad(4.02) ; 305$ (5.08); 337 (4.33) (Fig.S3). IR $\left(\mathrm{cm}^{-1}\right): \mathrm{v}(\mathrm{OH})$ 3310; $\mathrm{v}(\mathrm{NH})$ 3396; $\mathrm{v}(\mathrm{CH})$ aliphatic 2961, 2842; $\mathrm{v}(\mathrm{C}-\mathrm{O})$ 1163; $\mathrm{v}(\mathrm{C}-\mathrm{N})$ 974; $\mathrm{v}(\mathrm{C}-\mathrm{S})$ 859; $\mathrm{v}\left(\mathrm{C}=\mathrm{N}^{1}\right)$ 1627; $\mathrm{v}\left(\mathrm{C}=\mathrm{N}^{2}\right) 1599$ (Fig.S9). ${ }^{1} \mathrm{H}-\mathrm{NMR}$ (ppm): $12.12,11.64(\mathrm{~s}, \mathrm{i}: 3 / 1,1 \mathrm{H},-\mathrm{OH}) ; 8.33,8.31$ (s, $\left.\mathrm{i}: 2 / 1,1 \mathrm{H}, \mathrm{CH}=\mathrm{N}^{1}\right) ; 7.11(\mathrm{~m}, 1 \mathrm{H}, d) ; 6.45(\mathrm{~m}$, $2 \mathrm{H}, \mathrm{a}, \mathrm{c}) ; 5.43,4.47\left(\mathrm{q}, \mathrm{i}: 1 / 2,1 \mathrm{H}, \mathrm{N}^{4} H\right) ; 3.78$ $\left(\mathrm{s}, 3 \mathrm{H},-\mathrm{OCH}_{3}\right) ; 2.99,2.93\left(\mathrm{~d}, \mathrm{i}: 3 / 1,3 \mathrm{H}, \mathrm{N}^{4}-\right.$ $\left.\mathrm{CH}_{3}\right) ; 3.04,2.79\left(\mathrm{t}, 2 \mathrm{H}, \mathrm{S}-\mathrm{C}^{1} \mathrm{H}_{2}-\right) ; 1.70(\mathrm{~m}, 2 \mathrm{H}$, $\left.-\mathrm{C}^{2} \mathrm{H}_{2}\right) ; 1.01$ (t, 3H, $\left.-\mathrm{C}^{3} \mathrm{H}_{3}\right)$ (Fig.S17). ${ }^{13} \mathrm{C}-\mathrm{NMR}$ $(\mathrm{ppm}): 164.65 \quad(=\mathrm{CN}-\mathrm{S}) ; 163.29 \quad(-\mathrm{CH}=\mathrm{N})$, 158.96, 157.51, 134.48, 115.22, 108.92, 103.86 (aromatics); $58.04\left(-\mathrm{OCH}_{3}\right) ; 33.26$ (S$\left.\mathrm{CH}_{2}^{-}\right) ; 25.39\left(-\mathrm{CH}_{2}^{-}\right) ; 16.18\left(-\mathrm{CH}_{3}\right) ; 35.08(\mathrm{~N}-$ $\mathrm{CH}_{3}$ ) (Fig.S25).

L4: Yellow, m.p. 174.8-175.4 ${ }^{\circ} \mathrm{C}$, yield $79 \%$. Anal. Calc. for $\mathrm{C}_{14} \mathrm{H}_{21} \mathrm{~N}_{3} \mathrm{O}_{2} \mathrm{~S}(295.40 \mathrm{~g} / \mathrm{mol})$ : C, 56.92; H, 7.17; N, 14.22; S, 10.85. Found: C, $56.85 ; \mathrm{H}, 7.32 ; \mathrm{N}, 14.16 ; \mathrm{S}, 10.53 \%$. UV-Vis $(\mathrm{nm}(\log \varepsilon)): 243$ (4.16); 296 (4.23); 308 (4.25); 345 (4.27) (Fig.S4). IR $\left(\mathrm{cm}^{-1}\right): \mathrm{v}(\mathrm{OH})$

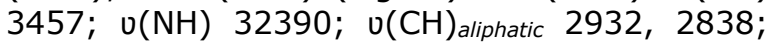
$\mathrm{v}(\mathrm{C}-\mathrm{O})$ 1187; $\mathrm{u}(\mathrm{C}-\mathrm{N})$ 959; $\mathrm{v}(\mathrm{C}-\mathrm{S})$ 854; $\mathrm{v}\left(\mathrm{C}=\mathrm{N}^{1}\right)$ 1627; $\mathrm{v}\left(\mathrm{C}=\mathrm{N}^{2}\right) 1603$ (Fig.S11). ${ }^{1} \mathrm{H}-\mathrm{NMR}$ (ppm): $12.12,11.69(\mathrm{~s}, \mathrm{i}: 2 / 1,1 \mathrm{H},-\mathrm{OH}) ; 8.33,8.32(\mathrm{~s}$, $\left.\mathrm{i}: 1 / 2,1 \mathrm{H}, \mathrm{CH}=\mathrm{N}^{1}\right) ; 7.12(\mathrm{~m}, 1 \mathrm{H}, d) ; 6.46(\mathrm{~m}$, $2 \mathrm{H}, \mathrm{a}, \mathrm{c}) ; 5.40,4.25\left(\mathrm{t}, \mathrm{i}: 1 / 2,1 \mathrm{H}, \mathrm{N}^{4} H\right) ; 3.80$ $\left(\mathrm{s}, 3 \mathrm{H},-\mathrm{OCH}_{3}\right) ; 3.45,3.32\left(\mathrm{~m}, \mathrm{i}: 2 / 1,2 \mathrm{H}, \mathrm{N}^{4}-\right.$ $\left.\mathrm{CH}_{2}\right) ; 1.25,1.21\left(\mathrm{t}, \mathrm{i}: 2 / 1,3 \mathrm{H},-\mathrm{CH}_{3}\right) ; 3.05$, $2.81\left(\mathrm{t}, 2 \mathrm{H}, \mathrm{S}-\mathrm{C}^{1} \mathrm{H}_{2}-\right) ; 1.72\left(\mathrm{~m}, 2 \mathrm{H},-\mathrm{C}^{2} \mathrm{H}_{2}\right)$; $1.03\left(\mathrm{t}, 3 \mathrm{H},-\mathrm{C}^{3} \mathrm{H}_{3}\right)$ (Fig.S19). ${ }^{13} \mathrm{C}-\mathrm{NMR}$ (ppm): $164.59(=\mathrm{CN}-\mathrm{S}) ; 163.28(-\mathrm{CH}=\mathrm{N}), 158.86$, 157.39, 134.47, 115.22, 108.98, 103.94 (aromatics); $58.04\left(-\mathrm{OCH}_{3}\right) ; 35.18\left(\mathrm{~S}-\mathrm{CH}_{2}^{-}\right)$; $25.51\left(-\mathrm{CH}_{2}^{-}\right) ; 16.10\left(-\mathrm{CH}_{3}\right) ; 41.12\left(\mathrm{~N}-\mathrm{CH}_{2}^{-}\right)$; $17.30\left(-\mathrm{CH}_{3}\right)$ (Fig.S27).

\section{Synthesis of Complexes}

The new complexes were prepared using literature method with small modifications (25, 26) (Figure 1). The synthesis procedure of complexes is the same as for complex $\mathbf{1}$, which is given as an example and has the IUPAC name cis-dioxo-(S-allyl-N $\mathrm{N}^{1}$-2-hydroxy-4methoxybenzylidene thiosemicarbazonato) $\left(\mathrm{N}, \mathrm{N}^{\prime}, \mathrm{O}\right)$-methanol-molybdenum(VI). 0.26 gram 
(1 millimole) gram of $\mathrm{N}^{1}$-2-hydroxy-4methoxybenzylidene thiosemicarbazone was dissolved in 3 milliliters of methanol with heating. 0.32 gram (1 millimole) of bis(acetylacetonato)dioxomolybdenum(VI) was dissolved in 2 milliliters of methanol and added dropwise to the reaction medium. The mixture was blended at about $45^{\circ} \mathrm{C}$ for one and a half hours. The red product was filtered and washed with a few drops of methanol. The product was recrystallized in methanol and obtained pure for use in characterization. The colors, melting points $\left({ }^{\circ} \mathrm{C}\right), \%$ yields, elemental analysis, UVVis, IR, ${ }^{1} \mathrm{H}-\mathrm{NMR}$ and ${ }^{13} \mathrm{C}-\mathrm{NMR}$ data of the complexes are given below:

1: Red, m.p. 215.8-216.4 ${ }^{\circ} \mathrm{C}$, yield $47 \%$. Anal. Calc. for $\mathrm{C}_{13} \mathrm{H}_{17} \mathrm{MoN}_{3} \mathrm{O}_{5} \mathrm{~S}$ (423.29 $\left.\mathrm{g} / \mathrm{mol}\right)$ : C, 36.89; H, 4.05; N, 9.93; S, 7.58. Found: C, $36.99 ; \mathrm{H}, 3.91 ; \mathrm{N}, 9.77 ; \mathrm{S}, 7.70 \%$. UV-Vis (nm $(\log \varepsilon)): 254$ (4.35); 302 (4.35); 420 (3.84) (Fig.S1). IR $\left(\mathrm{cm}^{-1}\right): \mathrm{v}(\mathrm{OH}) 3376 ; \mathrm{v}(\mathrm{NH}) 3228$; $\mathrm{U}(\mathrm{CH})$ aliphatic 2984, 2843; U(C-O) 1187; U(C-N) 1013; $\mathrm{v}(\mathrm{C}-\mathrm{S})$ 846; $\mathrm{v}\left(\mathrm{C}=\mathrm{N}^{1}\right)$ 1540; $\mathrm{u}\left(\mathrm{C}=\mathrm{N}^{2}\right)$ 1498; Us, $\mathrm{U}_{\mathrm{as}}\left(\mathrm{MoO}_{2}\right)$ 929, 901 (Fig.S6). ${ }^{1} \mathrm{H}-\mathrm{NMR}$ (ppm): $8.73\left(\mathrm{~s}, 1 \mathrm{H}, \mathrm{CH}=\mathrm{N}^{1}\right) ; 7.43(\mathrm{~d}, 1 \mathrm{H}, d)$; $6.71(\mathrm{dd}, 1 \mathrm{H}, \mathrm{c}) ; 6.65(\mathrm{~d}, 1 \mathrm{H}, \mathrm{a}) ; 6.90(\mathrm{~s}, 1 \mathrm{H}$, $\left.\mathrm{N}^{4} \mathrm{H}\right) ; 3.86\left(\mathrm{~s}, 3 \mathrm{H},-\mathrm{OCH}_{3}\right) ; 3.77(\mathrm{~d}, 2 \mathrm{H}, \mathrm{S}-$ $\left.\mathrm{C}^{1} \mathrm{H}_{2}^{-}\right)$; $5.98\left(\mathrm{~m}, 1 \mathrm{H},-\mathrm{C}^{2} \mathrm{H}=\right)$; 5.33, $5.20(\mathrm{~d}, 2 \mathrm{H}$, $\left.=\mathrm{C}^{3} \mathrm{H}_{2}\right) ; 3.48\left(\mathrm{~s}, 3 \mathrm{H}, \mathrm{O}-\mathrm{CH}_{3}\right)$ (Fig.S14). ${ }^{13} \mathrm{C}-\mathrm{NMR}$ (ppm): 171.35 (=CN-S); $168.90(-\mathrm{CH}=\mathrm{N})$, 165.44, 158.84, 135.42, 115.55, 114.42, 105.55 (aromatics); $58.52\left(-\mathrm{OCH}_{3}\right) ; 37.68$ (S$\left.\mathrm{CH}_{2}{ }^{-}\right) ; 136.84(-\mathrm{CH}=) ; 121.62\left(=\mathrm{CH}_{2}\right) ; 56.15$ $\left(\mathrm{OH}-\mathrm{CH}_{3}\right)$ (Fig.S22).

2: Red, m.p. $115.7-116.4^{\circ} \mathrm{C}$, yield $35 \%$. Anal. Calc. for $\mathrm{C}_{14} \mathrm{H}_{18} \mathrm{CIMON}_{3} \mathrm{O}_{4} \mathrm{~S}(455.76 \mathrm{~g} / \mathrm{mol})$ : C, 36.89; H, 3.98; N, 9.22; S, 7.04. Found: C, $36.72 ; \mathrm{H}, 3.80 ; \mathrm{N}, 9.37 ; \mathrm{S}, 7.17 \%$. UV-Vis (nm $(\log \varepsilon)): 252$ (4.34); 302 (4.34); 420 (3.84) (Fig.S2). IR $\left(\mathrm{cm}^{-1}\right): \mathrm{v}(\mathrm{OH}) 3394 ; \mathrm{v}(\mathrm{CH})_{\text {aliphatic }}$ 2962, 2815; v(C-O) 1164; v(C-N) 1018; v(C-S) 833; $\mathrm{v}\left(\mathrm{C}=\mathrm{N}^{1}\right) \quad 1597 ; \quad \mathrm{v}\left(\mathrm{C}=\mathrm{N}^{2}\right) \quad 1566 ; \quad \mathrm{us}_{\mathrm{s}}$ $\mathrm{U}_{\mathrm{as}}\left(\mathrm{MoO}_{2}\right)$ 909, 882 (Fig.S8). ${ }^{1} \mathrm{H}-\mathrm{NMR}$ (ppm): $8.64\left(\mathrm{~s}, 1 \mathrm{H}, \mathrm{CH}=\mathrm{N}^{1}\right) ; 7.45(\mathrm{~m}, 2 \mathrm{H}, b, d) ; 7.06$ $(\mathrm{m}, 1 \mathrm{H}, \mathrm{a}) ; 3.84\left(\mathrm{~m}, 2 \mathrm{H}, \mathrm{N}^{4}-\mathrm{CH}_{2}\right) ; 1.32(\mathrm{t}, 3 \mathrm{H}$, $\left.-\mathrm{CH}_{3}\right) ; 3.84\left(\mathrm{~m}, 2 \mathrm{H}, \mathrm{S}-\mathrm{C}^{1} \mathrm{H}_{2}-\right)$; $5.98(\mathrm{~m}, 1 \mathrm{H}$, $\left.\mathrm{C}^{2} \mathrm{H}=\right) ; 5.33,5.20\left(\mathrm{~d}, 2 \mathrm{H},=\mathrm{C}^{3} \mathrm{H}_{2}\right) ; 3.47(\mathrm{~s}, 3 \mathrm{H}$, $\left.\mathrm{O}-\mathrm{CH}_{3}\right)$ (Fig.S16). ${ }^{13} \mathrm{C}-\mathrm{NMR} \quad$ (ppm): 175.24 $(=\mathrm{CN}-\mathrm{S}) ; 161.37(-\mathrm{CH}=\mathrm{N}), 156.26,135.39$, $133.88,129.63,123.28,123.20$ (aromatics); $37.51 \quad\left(\mathrm{~S}-\mathrm{CH}_{2}-\right) ; \quad 137.35 \quad(-\mathrm{CH}=) ; 121.69$ $\left(=\mathrm{CH}_{2}\right) ; 51.76\left(\mathrm{~N}-\mathrm{CH}_{2}-\right) ; 17.80\left(-\mathrm{CH}_{3}\right) ; 57.05$ $\left(\mathrm{OH}-\mathrm{CH}_{3}\right)(\mathrm{Fig} . \mathrm{S} 24)$.

3: Red, m.p. 200.1-200.8 ${ }^{\circ} \mathrm{C}$, yield $80 \%$. Anal. Calc. for $\mathrm{C}_{14} \mathrm{H}_{21} \mathrm{MoN}_{3} \mathrm{O}_{5} \mathrm{~S}(439.34 \mathrm{~g} / \mathrm{mol}): \mathrm{C}$, 38.27; H, 4.82; N, 9.56; S, 7.30. Found: C,
$38.41 ; H, 4.91 ; N, 9.73 ; \mathrm{S}, 7.45 \%$. UV-Vis (nm $(\log \varepsilon)): 254$ (4.32); 303 (4.33); 420 (3.78) (Fig.S3). IR $\left(\mathrm{cm}^{-1}\right): \mathrm{v}(\mathrm{OH}) 3325 ; \mathrm{v}(\mathrm{CH})_{\text {aliphatic }}$ 2961, 2869; v(C-O) 1152; v(C-N) 1022; v(C-S) 848; $\quad \mathrm{v}\left(\mathrm{C}=\mathrm{N}^{1}\right) \quad 1582 ; \quad \mathrm{v}\left(\mathrm{C}=\mathrm{N}^{2}\right) \quad 1539 ; \quad \mathrm{U}_{\mathrm{s}}$,

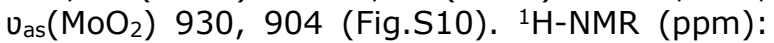
$8.64\left(\mathrm{~s}, 1 \mathrm{H}, \mathrm{CH}=\mathrm{N}^{1}\right) ; 7.37(\mathrm{~d}, 1 \mathrm{H}, d) ; 6.66(\mathrm{dd}$, $1 \mathrm{H}, \mathrm{c}) ; 6.60(\mathrm{~d}, 1 \mathrm{H}, \mathrm{a}) ; 3.85\left(\mathrm{~s}, 3 \mathrm{H},-\mathrm{OCH}_{3}\right)$; $3.52\left(\mathrm{~s}, 3 \mathrm{H}, \mathrm{N}^{4}-\mathrm{CH}_{3}\right) ; 3.11\left(\mathrm{t}, 2 \mathrm{H}, \mathrm{S}-\mathrm{C}^{1} \mathrm{H}_{2}-\right)$; $1.77\left(\mathrm{~m}, 2 \mathrm{H},-\mathrm{C}^{2} \mathrm{H}_{2}\right) ; 1.04\left(\mathrm{t}, 3 \mathrm{H},-\mathrm{C}^{3} \mathrm{H}_{3}\right) ; 3.48$ (d, 3H, O- $\mathrm{CH}_{3}$ ) (Fig.S18). ${ }^{13} \mathrm{C}-\mathrm{NMR}$ (ppm): $174.20(=\mathrm{CN}-\mathrm{S}) ; 168.79(-\mathrm{CH}=\mathrm{N}), 164.89$, $157.71,136.45,115.48,113.89,105.22$ (aromatics); $58.55\left(-\mathrm{OCH}_{3}\right) ; 36.73\left(\mathrm{~S}-\mathrm{CH}_{2}-\right)$; $25.19\left(-\mathrm{CH}_{2}-\right) ; 16.18\left(-\mathrm{CH}_{3}\right) ; 48.92\left(\mathrm{~N}-\mathrm{CH}_{3}\right)$; $53.56\left(\mathrm{OH}-\mathrm{CH}_{3}\right)$ (Fig.S26).

4: Red, m.p. 150.4-151.2 ${ }^{\circ} \mathrm{C}$, yield $56 \%$. Anal. Calc. for $\mathrm{C}_{15} \mathrm{H}_{23} \mathrm{MoN}_{3} \mathrm{O}_{5} \mathrm{~S} \quad(453.36 \mathrm{~g} / \mathrm{mol}): \mathrm{C}$, 39.74; H, 5.11; N, 9.27 S, 7.07. Found: C, $39.59 ; \mathrm{H}, 5.31 ; \mathrm{N}, 8.48 ; \mathrm{S}, 7.24 \%$. UV-Vis (nm $(\log \varepsilon)): 252$ (4.29); 303 (4.30); 422 (3.76) (Fig.S4). IR $\left(\mathrm{cm}^{-1}\right): \mathrm{v}(\mathrm{OH}) 3312 ; \mathrm{v}(\mathrm{CH})_{\text {aliphatic }}$ 2967, 2872; v(C-O) 1175; v(C-N) 1023; v(C-S) 858; $\quad \mathrm{v}\left(\mathrm{C}=\mathrm{N}^{1}\right) \quad 1576 ; \quad \mathrm{v}\left(\mathrm{C}=\mathrm{N}^{2}\right) \quad 1531 ; \quad \mathrm{U}_{\mathrm{s}}$, $\mathrm{U}_{\mathrm{as}}\left(\mathrm{MoO}_{2}\right)$ 933, 907 (Fig.S12). ${ }^{1} \mathrm{H}-\mathrm{NMR}$ (ppm): $8.64\left(\mathrm{~s}, 1 \mathrm{H}, \mathrm{CH}=\mathrm{N}^{1}\right) ; 7.37(\mathrm{~d}, 1 \mathrm{H}, d) ; 6.66$ (dd, $1 \mathrm{H}, c) ; 6.62(\mathrm{~d}, 1 \mathrm{H}, \mathrm{a}) ; 3.85\left(\mathrm{~s}, 3 \mathrm{H},-\mathrm{OCH}_{3}\right)$; $3.82\left(\mathrm{q}, 2 \mathrm{H}, \mathrm{N}^{4}-\mathrm{CH}_{2}\right) ; 1.31\left(\mathrm{t}, 3 \mathrm{H},-\mathrm{CH}_{3}\right) ; 3.12$ $\left(\mathrm{t}, 2 \mathrm{H}, \mathrm{S}-\mathrm{C}^{1} \mathrm{H}_{2}-\right) ; 1.77\left(\mathrm{~m}, 2 \mathrm{H},-\mathrm{C}^{2} \mathrm{H}_{2}\right) ; 1.04(\mathrm{t}$, $\left.3 \mathrm{H},-\mathrm{C}^{3} \mathrm{H}_{3}\right) ; 3.47$ (d, 3H, O- $\mathrm{CH}_{3}$ ) (Fig.S2O). ${ }^{13} \mathrm{C}-$ NMR (ppm): $173.02(=\mathrm{CN}-\mathrm{S}) ; 168.86(-\mathrm{CH}=\mathrm{N})$, 164.97, 157.66, 136.40, 115.51, 113.81, 105.32 (aromatics); $58.47\left(-\mathrm{OCH}_{3}\right) ; 36.68$ (S$\left.\mathrm{CH}_{2}^{-}\right) ; 25.24\left(-\mathrm{CH}_{2}^{-}\right) ; 16.14\left(-\mathrm{CH}_{3}\right) ; 53.51$ (N$\left.\mathrm{CH}_{2}{ }^{-}\right) ; 17.57\left(-\mathrm{CH}_{3}\right) ; 56.66\left(\mathrm{OH}-\mathrm{CH}_{3}\right)$ (Fig.S28).

\section{X-ray Crystallography}

The suitable crystals of $\mathbf{1}$ and $\mathbf{2}$ were obtained by slow evaporation of methanolic solutions. Red crystals of $\mathbf{1}\left(\mathrm{C}_{13} \mathrm{H}_{17} \mathrm{MoN}_{3} \mathrm{O}_{5} \mathrm{~S}\right)$ and 2 $\left(\mathrm{C}_{14} \mathrm{H}_{18} \mathrm{CIMoN}_{3} \mathrm{O}_{4} \mathrm{~S}\right)$ having dimensions of $0.243 \times 0.11 \times 0.057 \mathrm{~mm}^{3}$ and $0.156 \times$ $0.097 \times 0.049 \mathrm{~mm}^{3}$ respectively were mounted on a glass fiber. The measurements were made on a Bruker APEX-II CCD imaging plate area detector with graphite monochromatic Mo-K a radiation $(\lambda=0.71073 \AA)$. The conditions of experimental details were summed up in Table 1. The crystal structures were solved and refined with SHELXTL (31). Other atoms except hydrogen were refined anisotropically. The structures were drawn with ORTEP-III program with $50 \%$ probability displacement ellipsoids (32). 


\section{RESULTS AND DISCUSSION}

\section{Some Characteristics of the Compounds}

The color of thiosemicarbazones is yellow. They can easily dissolve in common solvents like pyridine, dimethylformamide, dimethyl sulfoxide, and alcohols. The reaction of the thiosemicarbazones

bis(acetylacetonato)dioxomolybdenum(VI) in methanol gave diamagnetic $\left[\mathrm{MoO}_{2}(\mathrm{~L}) \mathrm{CH}_{3} \mathrm{OH}\right]$ complexes. These dioxomolybdenum(VI) complexes are dissolve in chloroform, dichloromethane, dimethyl sulfoxide and alcohols. They are stable in the air but lose their shine after a few months. They decompose and turn into black amorphous substances, when stirred for several hours at about 50-60 ${ }^{\circ} \mathrm{C}$ in solution.

The S-alkyl thiosemicarbazones were obtained in higher yields than dioxomolybdenum(VI) complexes. The 5-chloro derivative compounds ( $\mathbf{L}^{\mathbf{2}}$ and $\mathbf{2}$ ) were obtained with the lowest yield (62 and $35 \%$ ) and melting point (101.2 and $\left.115.7^{\circ} \mathrm{C}\right)$ in its group. In addition, the melting point of the complexes was higher than the corresponding ligands except $\mathbf{L}^{\mathbf{4}}$ and $\mathbf{4}$.

\section{Crystallographic Studies}

The crystals of $\mathbf{1}\left[\mathrm{MoO}_{2} \mathrm{~L}^{1} \mathrm{CH}_{3} \mathrm{OH}\right]$ and 2 $\left[\mathrm{MoO}_{2} \mathrm{~L}^{2} \mathrm{CH}_{3} \mathrm{OH}\right]$ were obtained by slow evaporation of methanolic solutions. To confirm the certain structure of the dioxomolybdenum(VI) complexes, X-ray crystallographic analysis have been carried out for $\mathbf{1}$ and $\mathbf{2}$. The ORTEP diagrams with the atoms labeled were presented in Figures 2 and 3 , while the network formation and molecular packing arrangement are given in Figures 4 and 5. The parameters of refinement and some details of crystallographic data were summed up in Table 1 . The molybdenum centered bond lengths and angles were given in Table 2. Also, Table 3 shows the intermolecular $\mathrm{H}$-bond interactions. Complex 1, $(0.243 \times 0.11 \times 0.057$ $\mathrm{mm}^{3}$ ) crystallized in the monoclinic $\mathrm{P} 2{ }_{1} / \mathrm{c}$ space group with $Z=4$, while $2(0.156 \times 0.097 \times 0.049$ $\mathrm{mm}^{3}$ ) crystallized within triclinic crystal system with $\mathrm{P}-1$ space group $(Z=2)$. The angular values of $\mathrm{O}(2)-\mathrm{Mo}(1) 1-\mathrm{O}(3)$ (for 1) and $\mathrm{O}(1)-\mathrm{Mo}-\mathrm{O}(4)$ (for 2) were 105.68 and 105.97 respectively, showing the cis- character of $\mathrm{MoO}_{2}{ }^{2+}(33,34)$. Considering the bonds and angles, it can be said that the geometric parameters of complexes are within the expected ranges when compared with similar cis-dioxomolybdenum centered complexes of thiosemicarbazones $(18,33)$. As results, the single crystal diffraction studies of dioxomolybdenum(VI) complexes revealed a distorted octahedral geometry. Accordingly, the thiosemicarbazones behaved as ONN tridentate by binding to $\mathrm{cis}-\mathrm{MoO}_{2}{ }^{2+}$ with the oxygen atom of aromatic moiety, the nitrogen atom of $\mathrm{CH}=\mathrm{N}^{1}$ and the thioamide group. Methanol molecule which reactions were carried out behaved as coligand and it occupied the sixth coordination site of molybdenum center (35). 

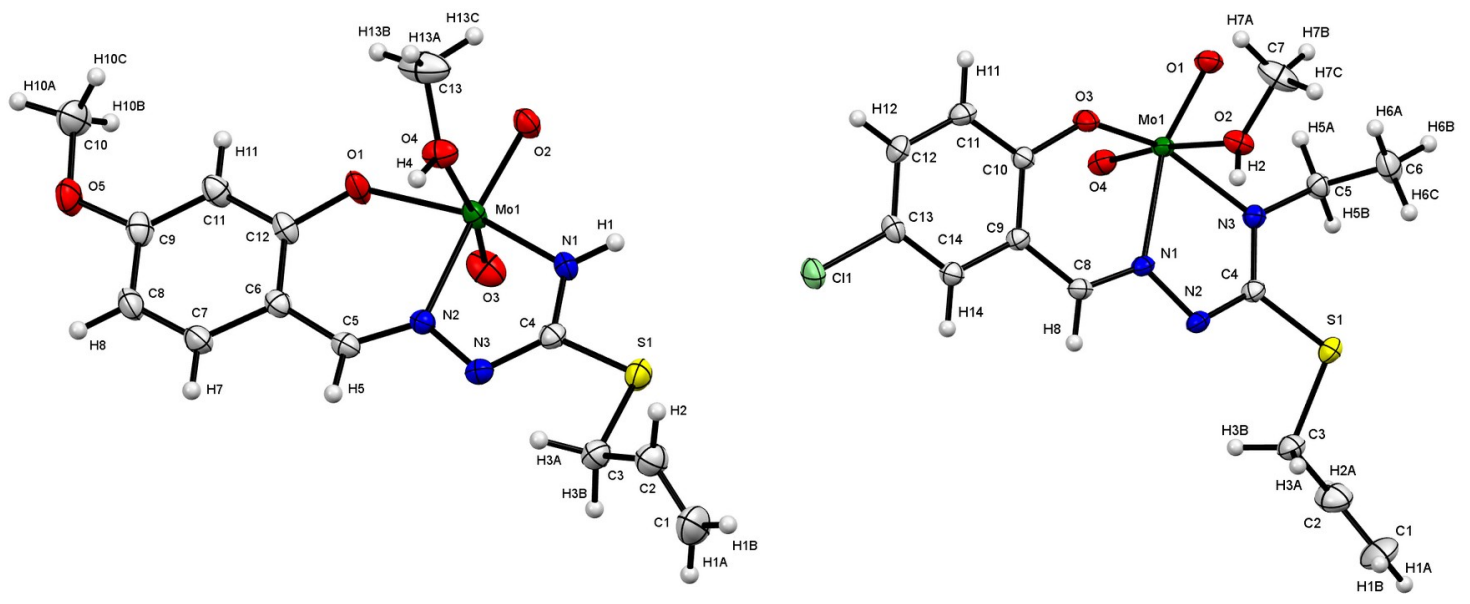

Figure 2: ORTEP view of cis-dioxo-(S-allyl-N $\mathrm{N}^{1}-2-$ Figure 3: ORTEP view of cis-dioxo-(S-allyl- $\mathrm{N}^{1}-2-$ hydroxy-4-methoxybenzylidenehydroxy-5-chlorobenzylidene- $\mathrm{N}^{4}$-ethyl thiosemicarbazonato) $\left(\mathrm{O}, \mathrm{N}, \mathrm{N}^{\prime}\right)$-methanolmolybdenum(VI) (1) thiosemicarbazonato) $\left(\mathrm{O}, \mathrm{N}, \mathrm{N}^{\prime}\right)$-methanolmolybdenum(VI) (2)

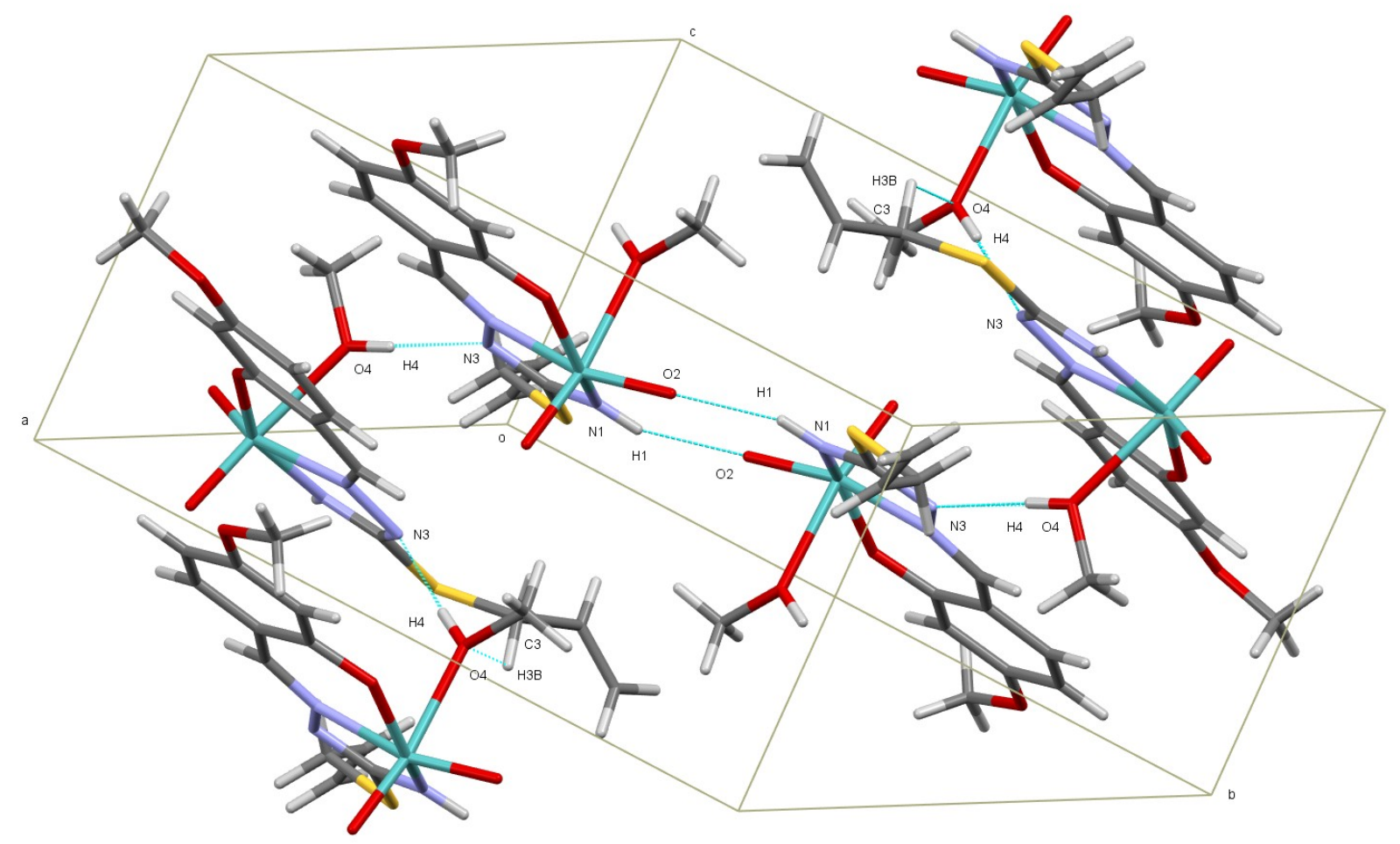

Figure 4: The packing diagram and hydrogen bonding network of complex $\mathbf{1}$. 


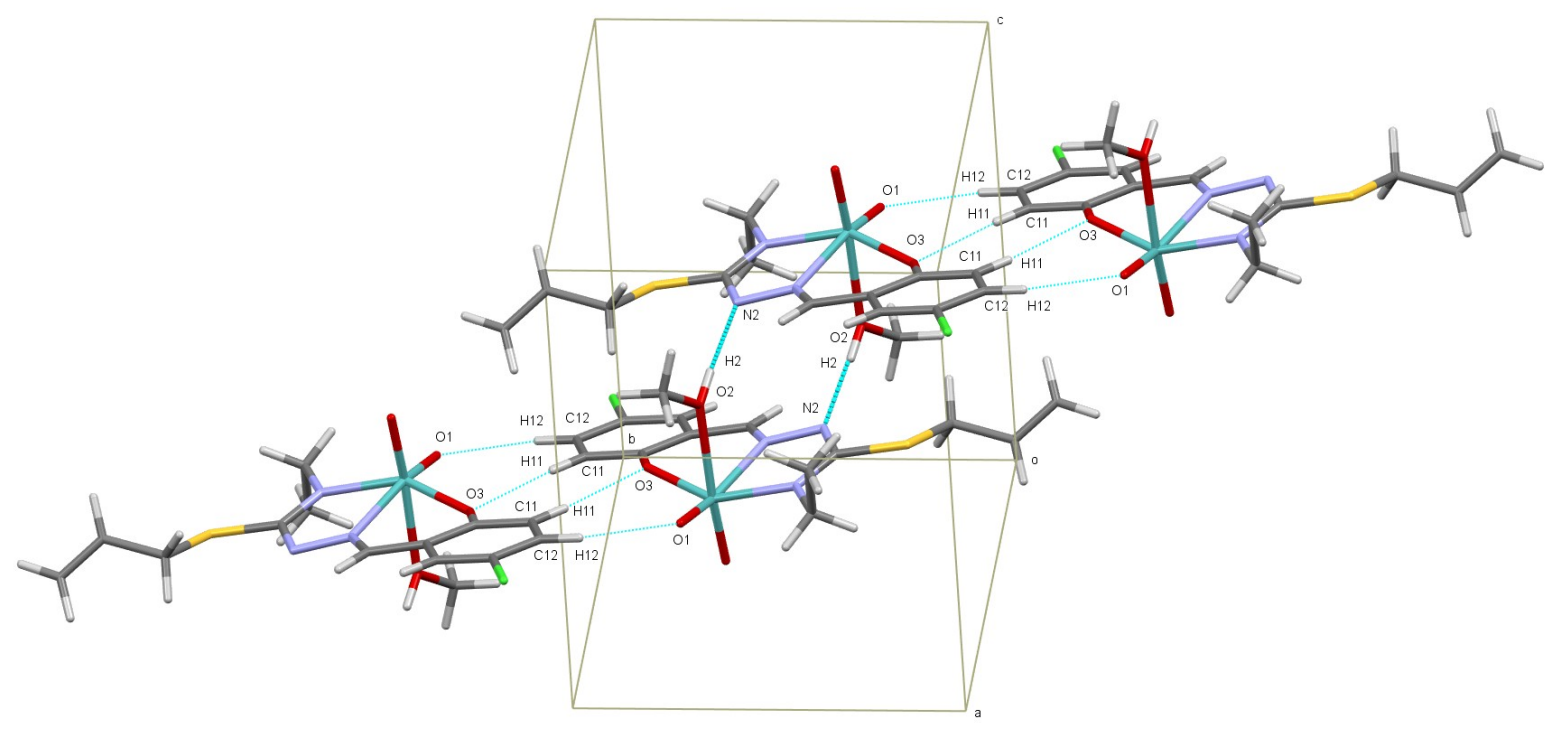

Figure 5: The packing diagram and hydrogen bonding network of complex $\mathbf{2}$.

Table 1: The structure refinements and crystal data for $\mathbf{1}$ and $\mathbf{2}$.

\section{Parameters \\ CCDC deposition no. \\ Chemical formula \\ Formula weight $\left(\mathrm{g} \cdot \mathrm{mol}^{-1}\right)$ \\ Temperature $(K)$ \\ Wavelength $(\AA)$ \\ Crystal system \\ Space group \\ Unit cell parameters}
$a, b, c(\AA)$
$\alpha, \beta, \gamma\left(^{\circ}\right)$

Volume $\left(\AA^{3}\right)$

Z

$D_{\text {calc }}\left(\mathrm{g} \cdot \mathrm{cm}^{-3}\right)$

Absorption correction

$\mathrm{F}(000)$

Crystal size $\left(\mathrm{mm}^{3}\right)$

Diffractometer/measurement method

Index ranges $h, k, I$

$2 \theta$ Range for data collection $\left({ }^{\circ}\right)$

Reflections collected

Independent reflections

Refinement method

$\mathbf{1}$
2104647
$\mathrm{C}_{13} \mathrm{H}_{17} \mathrm{MoN}_{3} \mathrm{O}_{5} \mathrm{~S}$
423.29
173.01
0.71073
monoclinic
$\mathrm{P} 2_{1} / \mathrm{c}$

$577(6), 17.124(8)$,
$7.528(3)$
90.000
$900(6)$,
$1713.6(13)$

4

1.641

multi-scan

856.0

$0.243 \times 0.111 \times 0.057$

$-17<=\mathrm{h}<=17$,

$-22<=\mathrm{k}<=22$,

$-9<=1<=9$

$3.064<2 \theta<54.966$

23095

3916

$\left[R_{\text {int }}=0.0452, R_{\text {sigma }}=\right.$ $0.0311]$

Full-matrix least-squares

\section{6}

$\mathrm{C}_{14} \mathrm{H}_{18} \mathrm{ClMoN}_{3} \mathrm{O}_{4} \mathrm{~S}$

455.76

173.01

0.71073

triclinic

P-1
$8.7870(7), 10.7115(9)$,
$10.8189(9)$
$100.1090(10), 105.6400(10)$,
$108.7600(10)$
$889.27(13)$
2
1.702
multi-scan
460.0
$0.156 \times 0.097 \times 0.049$

$-11 \leq \mathrm{h} \leq 11$,

$-13 \leq \mathrm{k} \leq 13$,

$-14 \leq \mathrm{I} \leq 14$

$4.082<2 \theta<54.95$

13504

4072

$\left[R_{\text {int }}=0.0269, R_{\text {sigma }}=\right.$ $0.0277]$

Full-matrix least-squares on $\mathrm{F}^{2}$ 


\begin{tabular}{lcc}
\hline Parameters & $\mathbf{1}$ & $\mathbf{2}$ \\
\hline Data/restraints/parameters & ${\text { on } \mathrm{F}^{2}}$ & \\
Goodness-of-fit on F & $3916 / 0 / 211$ & $4072 / 3 / 230$ \\
Final R indexes [I $>=2 \sigma(\mathrm{I})]$ & 1.042 & 1.037 \\
R indices (all data) & $\mathrm{R}_{1}=0.0256, \mathrm{wR}_{2}=0.0581$ & $\mathrm{R}_{1}=0.0232, \mathrm{wR}_{2}=0.0521$ \\
\hline
\end{tabular}

Table 2: The molybdenum centered bond lengths $(\AA)$ and angles $\left({ }^{\circ}\right)$ for complex $\mathbf{1}$ and 2.

\begin{tabular}{cllll}
\hline Complex & Atoms & Distances & Atoms & Angles \\
\hline \multirow{4}{*}{$\mathbf{1}$} & $\mathrm{Mo}(1)-\mathrm{N}(1)$ & $2.0230(19)$ & $\mathrm{O}(4)-\mathrm{Mo}(1)-\mathrm{N}(1)$ & $81.85(7)$ \\
& $\mathrm{Mo}(1)-\mathrm{N}(2)$ & $2.229(2)$ & $\mathrm{O}(2)-\mathrm{Mo}(1)-\mathrm{N}(1)$ & $93.63(8)$ \\
& $\mathrm{Mo}(1)-\mathrm{O}(1)$ & $1.9373(17)$ & $\mathrm{O}(3)-\mathrm{Mo}(1)-\mathrm{N}(1)$ & $100.48(9)$ \\
& $\mathrm{Mo}(1)-\mathrm{O}(4)$ & $2.3513(17)$ & $\mathrm{O}(2)-\mathrm{Mo}(1)-\mathrm{O}(4)$ & $83.61(7)$ \\
& $\mathrm{Mo}(1)-\mathrm{O}(2)$ & $1.7110(16)$ & $\mathrm{O}(3)-\mathrm{Mo}(1)-\mathrm{O}(4)$ & $171.13(7)$ \\
& $\mathrm{Mo}(1)-\mathrm{O}(3)$ & $1.6954(18)$ & $\mathrm{O}(2)-\mathrm{Mo}(1)-\mathrm{O}(3)$ & $105.68(8)$ \\
\hline \multirow{4}{*}{$\mathbf{2}$} & $\mathrm{Mo}(1)-\mathrm{N}(3)$ & $2.0695(16)$ & $\mathrm{O}(2)-\mathrm{Mo}(1)-\mathrm{N}(3)$ & $79.64(6)$ \\
& $\mathrm{Mo}(1)-\mathrm{N}(1)$ & $2.2422(16)$ & $\mathrm{O}(1)-\mathrm{Mo}(1)-\mathrm{N}(3)$ & $95.43(6)$ \\
& $\mathrm{Mo}(1)-\mathrm{O}(3)$ & $1.9476(13)$ & $\mathrm{O}(4)-\mathrm{Mo}(1)-\mathrm{N}(3)$ & $99.84(7)$ \\
& $\mathrm{Mo}(1)-\mathrm{O}(2)$ & $2.3515(14)$ & $\mathrm{O}(2)-\mathrm{Mo}(1)-\mathrm{O}(1)$ & $82.92(6)$ \\
& $\mathrm{Mo}(1)-\mathrm{O}(1)$ & $1.7076(14)$ & $\mathrm{O}(4)-\mathrm{Mo}(1)-\mathrm{O}(2)$ & $171.09(6)$ \\
& $\mathrm{Mo}(1)-\mathrm{O}(4)$ & $1.6948(15)$ & $\mathrm{O}(1)-\mathrm{Mo}(1)-\mathrm{O}(4)$ & $105.96(7)$ \\
\hline
\end{tabular}


Table 3: Intermolecular interaction parameters $\left(\AA\right.$ and $\left.{ }^{\circ}\right)$ of complex $\mathbf{1}$ and $\mathbf{2}$.

\begin{tabular}{|c|c|c|c|c|c|}
\hline Complex & $D-H \cdots A$ & $\mathrm{D}-\mathrm{H}$ & $H \cdots A$ & D...A & $D-H \cdots A$ \\
\hline \multirow{3}{*}{1} & $\mathrm{~N} 1-\mathrm{H} 1 \cdots \mathrm{O} 2$ & 0.88 & 2.158 & $3.003(3)$ & 160.8 \\
\hline & $\mathrm{O} 4-\mathrm{H} 4 \cdots \mathrm{N} 3$ & 0.84 & 1.957 & $2.796(3)$ & 176.7 \\
\hline & $\mathrm{C} 3-\mathrm{H} 3 \mathrm{~B} \cdots \mathrm{O} 4$ & 0.99 & 2.713 & $3.221(3)$ & 112.32 \\
\hline \multirow{3}{*}{2} & $\mathrm{C} 12-\mathrm{H} 12 \cdots \mathrm{O} 1$ & 0.950 & 2.705 & $3.336(3)$ & 124.6 \\
\hline & $\mathrm{C} 11-\mathrm{H} 11 \cdots \mathrm{O} 3$ & 0.950 & 2.694 & $3.639(3)$ & 172.8 \\
\hline & $\mathrm{O} 2-\mathrm{H} 2 \cdots \mathrm{N} 2$ & $0.86(2)$ & $1.85(2)$ & $2.707(3)$ & $176(2)$ \\
\hline
\end{tabular}

\section{UV-Vis Spectra}

In order to follow the formation of the complex and to determine at which wavelengths the electronic transitions are, the UV-Vis absorption bands of the compounds were recorded in chloroform solution $\left(3 \times 10^{-5} \mathrm{M}\right)$ between 200 and $800 \mathrm{~nm}$. In the spectra of the $\mathbf{L}^{\mathbf{1}}-\mathbf{L}^{\mathbf{4}}$ the bands recorded at $240-243 \mathrm{~nm}$ belong to $\Pi \rightarrow \Pi^{*}$ transitions of 4-methoxy and 5-chloro substituted aromatic rings $(33,35)$. The bands at about 295, 305 (as shoulders) and 336-345 (broad) $\mathrm{nm}$ can attributed as $\mathrm{n} \rightarrow \Pi^{*}$ transitions originating from the non-bonding electrons of thioamide and imine nitrogen atoms. In the spectra of 1-4, the absorption bands seen at 252-254 $\mathrm{nm}$ and $302-303 \mathrm{~nm}$ were related to the transitions of $n \rightarrow \Pi^{*}$ and $n \rightarrow \Pi^{*}$. The ligandmetal charge transfer transitions of the $\mathbf{1 - 4}$ were seen in range of $420-422 \mathrm{~nm}$. The forbidden transitions were not observed in the spectra of complexes since they have $4 d^{0}$ configuration $(12,27)$. The UV-Vis spectra of all compounds were given in the supplementary file (Figure S1-S4).

\section{IR Spectra}

In the IR spectra of $\mathbf{L}^{\mathbf{1}}-\mathbf{L}^{\mathbf{4}}$, the bands belonging to $\mathrm{v}(\mathrm{OH})$ were recorded at $3457-3310 \mathrm{~cm}^{-1}$. This band disappeared after complexation. Instead, bands belonging to the hydroxy group originating from methanol, which is coordinated to the metal center, were also observed at 3394-3276 $\mathrm{cm}^{-1}$. These values are lower than the $\mathrm{v}(\mathrm{OH})$ frequency value of non-coordinated of methanol molecule; $3682 \mathrm{~cm}^{-1}$ (36). This indicates that the methanol is coordinated. In the spectrum of $\mathbf{L}^{\mathbf{1}}$, the asymmetric and symmetric $v\left(\mathrm{NH}_{2}\right)$ vibration bands were recorded at 3414 and $3310 \mathrm{~cm}^{-1}$ respectively. The fact that this band was observed as a single band at $3228 \mathrm{~cm}^{-1}$ in the spectrum of $\mathbf{1}$ supports deprotonation of the ligand. Also, the $\mathrm{u}(\mathrm{NH})$ vibration of other ligands $\left(\mathbf{L}^{\mathbf{2}}-\mathbf{L}^{\mathbf{4}}\right)$, recorded at 3396-3228 $\mathrm{cm}^{-1}$, were disappeared by complexation. The band belonging to the $\mathrm{C}=\mathrm{N}^{2}$ group was recorded between 1602-1599 $\mathrm{cm}^{-1}$ in the spectra of thiosemicarbazones. After complexation, these bands shifted to lower frequencies as 1566-1498 $\mathrm{cm}^{-1}$. These situations support the ONN coordination formed by the removal of a hydrogen atom from the thioamide group. In addition, for all molybdenum complexes $(\mathbf{1}-\mathbf{4})$, the $v_{a s}$ and $v_{s}$ bands of $\mathrm{cis}-\mathrm{MoO}_{2}{ }^{2+}$ were recorded between 933-909 and 907-882 $\mathrm{cm}^{-1}(12,18)$. The IR spectra of all compounds were given in the supplementary file (Figure S5-S12).

\section{${ }^{1} \mathbf{H}-N M R$ Spectra}

In the ${ }^{1} \mathrm{H}-\mathrm{NMR}$ spectra of $\mathbf{L}^{\mathbf{1}}-\mathbf{L}^{\mathbf{4}}$, the expected isomeric peaks of phenolic hydrogen, azomethine $\left(\mathrm{CH}=\mathrm{N}^{1}\right), \mathrm{N}^{4} \mathrm{H}$ and also $\mathrm{N} / \mathrm{S}$-alkyl protons were recorded with different isomeric ratios. The signals of phenolic hydrogen belonging to the $2-\mathrm{OH}$ aldehyde appeared as two singlets between 12.12-11.41 ppm due to cis-trans isomerism. The absence of this peak in the spectra of 1-4 clearly proves the bonding of oxygen atom of aromatic moiety to the metal center. The syn-anti isomerism of the azomethine group, seen between 8.37-8.29 ppm, was not observed in the spectra of complexes (18). Besides, the shifting of these peaks to higher field indicates the coordination through azomethine group (35). In the spectrum of $\mathbf{L}^{\mathbf{1}}$ the band of the $\mathrm{N}^{4} \mathrm{H}_{2}$ was recorded in two proton integral at $5.08 \mathrm{ppm}$. When its corresponding complex 1 spectrum was examined, it was observed that this peak shifted to a lower area at $6.90 \mathrm{ppm}$, and was in a single proton integral. In the other complexes 2-4 spectra, there were no proton signals of $\mathrm{N}^{4} \mathrm{H}$ group. This situation supports that the thiosemicarbazones coordinate to the molybdenum center with amide nitrogen. When the spectra of all complexes (1-4) were examined, the $-\mathrm{CH}_{3}$ group protons of the methanol, coordinated as second ligand, were observed in the range of 3.48-3.47 ppm as singlets or doublets. The reason why the signal of the -OH group of methanol is not seen can be considered as the proton becoming more acidic due to intermolecular interactions $(12,33)$. The ${ }^{1} \mathrm{H}-\mathrm{NMR}$ spectra of all compounds were given in the supplementary file (Figures S13-S20). 


\section{${ }^{13}$ C-NMR Spectra}

In the ${ }^{13} \mathrm{C}$-NMR spectra of $\mathbf{L}^{\mathbf{1}} \mathbf{-} \mathbf{L}^{\mathbf{4}}$, the signal of the $=\mathrm{CN}-\mathrm{S}$ was observed in the lowest field between 164.26-165.35 ppm (37). When the spectra of complexes (1-4) were examined, it was observed that this signal shifted to about 10 ppm lower area. Also, the signals showed by azomethine nitrogen $(-\mathrm{CH}=\mathrm{N})$ for thiosemicarbazone ligands were recorded at the range 159.87-163.40 ppm. A slight lowfield shift in the azomethine resonance signal observed in complexes is due to the coordination of nitrogen atom. In addition, the carbon signals belonging to the $\mathrm{N}$-alkyl group $\left(\mathrm{N}-\mathrm{CH}_{3}\right.$ and $\left.\mathrm{N}-\mathrm{CH}_{2}^{-}\right)$were also shifted to the lower area due to the coordination of the thioamide nitrogen to the metal center. In all compounds except $\mathbf{L}^{\mathbf{2}}$ and $\mathbf{2}$, the signals belonging to the methoxy group was observed at approximately $58 \mathrm{ppm}$ (38). No significant change was observed with the complexation in the signals belonging to the methoxy and $\mathrm{S}$ alkyl groups. The peaks appearing at 53.56$57.05 \mathrm{ppm}$ in the complex spectra belong to the methanol molecule coordinated as second ligand to the dioxomolybdenum(VI) center. The ${ }^{13} \mathrm{C}-\mathrm{NMR}$ spectra of all compounds were given in the supplementary file (Figures S21-S28).

\section{CONCLUSION}

In this study, four dioxomolybdenum(VI) complexes were prepared with new thiosemicarbazone ligands having different substituents on the aromatic ring, the amide nitrogen and sulfur atoms to be used as models in future biological studies. The structural characterizations of all compounds were carried out by using elemental analysis, UV-Vis, IR, ${ }^{1} \mathrm{H}-$ NMR and ${ }^{13} \mathrm{C}-\mathrm{NMR}$ spectroscopies. The formation of dioxomolybdenum(VI) complexes was observed with the band recorded around $420 \mathrm{~nm}$ in the UV-Vis spectra, and the characteristic symmetric and asymmetric stretch bands recorded between 933-909 and 907-882 $\mathrm{cm}^{-1}$ belonging to the $\mathrm{MoO}_{2}{ }^{2+}$ group in the IR spectra. When the ${ }^{1} \mathrm{H}-\mathrm{NMR}$ spectra are examined, the disappearance of the signals belonging to the protons of the phenolic oxygen and thioamide nitrogen supports the coordination from these atoms to the metal center. In addition, when both the ${ }^{1} \mathrm{H}-\mathrm{NMR}$ and ${ }^{13} \mathrm{C}-\mathrm{NMR}$ spectra of the complexes were examined, the peaks of the methanol molecule were clearly observed. The crystal structure of two complexes $(\mathbf{1}, \mathbf{2})$ were confirmed by X-ray diffraction method. Crystal analysis of complexes indicated a distorted octahedral geometry. Comparison with complexes and previous reported similar molecules revealed that the bonds and angles of complexes are within the expected ranges. The crystallographic and spectroscopic data of the compounds clearly demonstrated the formation of ONN coordinated dioxomolybdenum(VI) complexes and binding to the metal center of the methanol molecule as second ligand.

\section{ACKNOWLEDGMENTS}

CCDC-2104647 for $1 \quad\left(\mathrm{C}_{13} \mathrm{H}_{17} \mathrm{MoN}_{3} \mathrm{O}_{5} \mathrm{~S}\right)$ and CCDC-2104646 for $2\left(\mathrm{C}_{14} \mathrm{H}_{18} \mathrm{ClMoN}_{3} \mathrm{O}_{4} \mathrm{~S}\right)$ contain the supplementary crystallographic data for this study. These data can be obtained free of charge from The Cambridge Crystallographic Data Centre via www.ccdc.cam.ac.uk/data request/cif

\section{REFERENCES}

1. Leovac V, Gerbeleu N, Canic V. Coordination compounds of cobalt (III), chromium (III), and vanadium (III) with salicylaldehyde Smethylthiosemicarbazone. Russ J Inorg Chem. $1982 ; 27: 514-7$.

2. Nehar OK, Mahboub R, Louhibi S, Roisnel T, Aissaoui M. New thiosemicarbazone Schiff base ligands: Synthesis, characterization, catecholase study and hemolytic activity. Journal of Molecular Structure. 2020 Mar; 1204:127566. <DOI $>$.

3. Behnisch R, Mietzsch F, Schmidt H. Chemical Studies on Thiosemicarbazones with Particular Reference to Antituberculous Acitivity. American review of tuberculosis. $1950 ; 61(1): 1-7$.

4. Plech $T$, Wujec M, Siwek A, Kosikowska U, Malm A. Synthesis and antimicrobial activity of thiosemicarbazides, s-triazoles and their Mannich bases bearing 3-chlorophenyl moiety. European Journal of Medicinal Chemistry. 2011 Jan;46(1):241-8. <DOI>

5. Kesel AJ. Broad-spectrum antiviral activity including human immunodeficiency and hepatitis $C$ viruses mediated by a novel retinoid thiosemicarbazone derivative. European Journal of Medicinal Chemistry. 2011 May;46(5):165664. $\leq \mathrm{DOI}>$

6. Li $\mathrm{MX}$, Chen $\mathrm{CL}$, Zhang $\mathrm{D}$, Niu JY, Ji BS. $\mathrm{Mn}(\mathrm{II}), \mathrm{Co}(\mathrm{II})$ and $\mathrm{Zn}$ (II) complexes with heterocyclic substituted thiosemicarbazones: Synthesis, characterization, X-ray crystal structures and antitumor comparison. European 
Journal of Medicinal Chemistry. 2010 Jul;45(7):3169-77. <DOI >.

7. Rodríguez-Argüelles MC, López-Silva EC, Sanmartín J, Pelagatti P, Zani F. Copper complexes of imidazole-2-, pyrrole-2- and indol-3-carbaldehyde thiosemicarbazones: Inhibitory activity against fungi and bacteria. Journal of Inorganic Biochemistry. 2005 Nov;99(11):2231-9. <DOI>.

8. Saswati, Dinda R, Schmiesing CS, Sinn E, Patil YP, Nethaji M, et al. Mixed-ligand nickel(II) thiosemicarbazone complexes: Synthesis, characterization and biological evaluation. Polyhedron. 2013 Feb;50(1):354-63. <DOI>.

9. Viñuelas-Zahínos $E$, Luna-Giles F, TorresGarcía P, Fernández-Calderón MC. Co(III), $\mathrm{Ni}(\mathrm{II}), \mathrm{Zn}(\mathrm{II})$ and $\mathrm{Cd}(\mathrm{II})$ complexes with 2acetyl-2-thiazoline thiosemicarbazone: Synthesis, characterization, X-ray structures and antibacterial activity. European Journal of Medicinal Chemistry. 2011 Jan;46(1):150-9. <DOI $>$.

10. Chen J, Huang Y, Liu G, Afrasiabi Z, Sinn E, Padhye $S$, et al. The cytotoxicity and mechanisms of 1,2-naphthoquinone thiosemicarbazone and its metal derivatives against MCF-7 human breast cancer cells. Toxicology and Applied Pharmacology. 2004 May;197(1):40-8. <DOI>.

11. Afrasiabi Z. Transition metal complexes of phenanthrenequinone thiosemicarbazone as potential anticancer agents: synthesis, structure, spectroscopy, electrochemistry and in vitro anticancer activity against human breast cancer cell-line, T47D. Journal of Inorganic Biochemistry. 2003 Jul 1;95(4):306-14. <DOI>.

12. Eğlence-Bakır $S$, Sacan $O$, Şahin $M$, Yanardag $R$, Ülküseven $B$. Dioxomolybdenum(VI) complexes with 3methoxy salicylidene-N-alkyl substituted thiosemicarbazones. Synthesis, characterization, enzyme inhibition and antioxidant activity. Journal of Molecular Structure. 2019 Oct;1194:35-41. <DOI>.

13. Eğlence-Bakır S. New nickel(II) complexes containing $\mathrm{N} 2 \mathrm{O} 2$ donor thiosemicarbazones: Synthesis, characterization and antioxidant properties. Journal of Molecular Structure. 2021 Dec; 1246:131121. <DOI>.
14. Bakkar MS, Siddiqi MY, Monshi MS. Preparation and Investegation of the Bonding Mode in the Complexes of $\mathrm{Pt}(\mathrm{II})$ with Thiosemicarbazone Ligands. Synthesis and Reactivity in Inorganic and Metal-Organic Chemistry. 2003 Jan 8;33(7):1157-69. <DOI>.

15. Ren P, Liu T, Qin J, Chen C. Synthesis, Crystal Structure, Spectroscopy and Calculated First-Order Molecular Hyperpolarizability of a New Square-pyramidal Complex Zn(DMABT) (acac) 2. Journal of Coordination Chemistry. 2003 Jan $1 ; 56(2): 125-32$. <DOI>.

16. Sankaraperumal A, Karthikeyan J, Shetty AN, Lakshmisundaram R. Nickel(II) complex of $\mathrm{p}$-[N,N-bis(2-chloroethyl)amino]benzaldehyde-

4-methyl thiosemicarbazone: Synthesis, structural characterization and biological application. Polyhedron. 2013 Feb;50(1):264-9. $\leq \mathrm{DOI}$.

17. Małecki JG, Maroń A, Serda M, Polański J. Ruthenium(II) carbonyl complexes with thiosemicarbazone ligands. Polyhedron. 2013 Jun;56:44-54. <DOI $>$.

18. Eğlence $S$, Şahin $M$, Özyürek $M$, Apak $R$, Ülküseven B. Dioxomolybdenum(VI) complexes of S-methyl-5-bromosalicylidene-N-alkyl substituted thiosemicarbazones: Synthesis, catalase inhibition and antioxidant activities. Inorganica Chimica Acta. 2018 Jan;469:495502. <DOI>.

19. Güveli Ş. Nickel(II)-PPh 3 complexes of substituted benzophenone thiosemicarbazones: Electrochemistry, structural analysis, and antioxidant properties. Journal of Coordination Chemistry. 2020 Jan 2;73(1):137-53. <DOI>.

20. Takjoo R, Hashemzadeh A, Rudbari HA, Nicolò F. Copper(II) and molybdenum(VI) complexes with 5-bromosalicylaldehyde S allylisothiosemicarbazone: Syntheses, characterizations and crystal structures. Journal of Coordination Chemistry. 2013 Jan $1 ; 66(2): 345-57$. <DOI>.

21. Yampolskaya M, Shova S, Gerbeleu N, Belskii V, Simonov YA. Synthesis And Structure Of [S-Methyl-N1, N4-Bis (Salicylidene) Thiosemicarbazide] Ferrochloride (III). Zhurnal Neorganıcheskoı Khımı. 1982;27(10):2551-7.

22. Şahin M, Eğlence-Bakır S, Alpay M, Alpay S, Özmerdivenli R, Ülküseven B. Effective copper(II) and nickel(II) complexes with N3O and ON3 thiosemicarbazidato ligands. 
Synthesis, structural analysis and in vitro cytotoxity on melanoma B16F10 cells. Inorganica Chimica Acta. 2020 Mar;502:119347. <DOI>.

23. Hille R. The Mononuclear Molybdenum Enzymes. Chem Rev. 1996 Jan 1;96(7):2757816. $\leq \mathrm{DOI}>$.

24. Hussein MA, Guan TS, Haque RA, Ahamed MBK, Majid AMSA. Synthesis and characterization of thiosemicarbazonato molybdenum(VI) complexes: In vitro DNA binding, cleavage, and antitumor activities. Polyhedron. 2015 Jan;85:93-103. <DOI>.

25. Duman S, Kızılcıklı İ, Koca A, Akkurt M, Ülküseven B. ONN-complexes of dioxomolybdenum(VI) with 2-hydroxy-1naphthaldehyde S-ethyl-4-H/phenylthiosemicarbazones: Crystal structure, electrochemistry and in situ spectroelectrochemistry. Polyhedron. 2010 Oct;29(15):2924-32. <DOI .

26. Duman S, Kizilcikli İ, Ülküseven B. Dioxomolybdenum(VI) Complexes of 5-Bromo/3,5-Dibromo-Salicylaldehyde 4-(H/C 6 H 5 )- S -Propyl-Thiosemicarbazones. Phosphorus, Sulfur, and Silicon and the Related Elements. 2015 Mar 4;190(3):342-51. <DOI>.

27. Moradi-Shoeili Z, Boghaei DM, Amini M, Bagherzadeh $M$, Notash $B$. New molybdenum(VI) complex with ONS-donor thiosemicarbazone ligand: Preparation, structural characterization, and catalytic applications in olefin epoxidation. Inorganic Chemistry Communications. 2013 Jan;27:2630. $\leq \mathrm{DOI}>$.

28. Vrdoljak V, Đilović I, Cindrić M, MatkovićČalogović D, Strukan N, Gojmerac-Ivšić A, et al. Synthesis, structure and properties of eight novel molybdenum(VI) complexes of the types: [MoO2LD] and [\{MoO2L $\} 2 D]$ ( $L=$ thiosemicarbazonato ligand, $D=N$-donor molecule). Polyhedron. 2009 Apr;28(5):95965. $\leq \mathrm{DOI}>$.

29. Takjoo R, Akbari A, Ahmadi M, Amiri Rudbari H, Bruno G. Synthesis, spectroscopy, DFT and crystal structure investigations of 3methoxy-2-hydroxybenzaldehyde Sethylisothiosemicarbazone and its $\mathrm{Ni}(\mathrm{II})$ and Mo(VI) complexes. Polyhedron. 2013 May;55:225-32.<DOI $>$.
30. Yamazaki C. The structure of isothiosemicarbazones. Canadian Journal of Chemistry. 1975;53(4):610-5.

31. Anonymous. SHELXTL, version 6.14. Bruker AXS Inc., Madison, WI; 2000.

32. Farrugia LJ. ORTEP -3 for Windows - a version of ORTEP -III with a Graphical User Interface (GUI). J Appl Crystallogr. 1997 Oct $1 ; 30(5): 565-565$. <DOI $>$.

33. Eğlence-Bakır $S$, Şahin $M$, Zahoor $M$, Dilmen-Portakal E, Ülküseven B. Synthesis and biological potentials of dioxomolybdenum(VI) complexes with ONS and ONN chelating thiosemicarbazones: DNA-binding, antioxidant and enzyme inhibition studies. Polyhedron. 2020 Nov; 190:114754. <DOI>.

34. Kaya Y, Erçağ A, Kaya K. Synthesis, characterization and antioxidant activities of dioxomolybdenum(VI) complexes of new Schiff bases derived from substituted benzophenones. Journal of Coordination Chemistry. 2018 Oct $18 ; 71(20): 3364-80$. $\leq$ DOI $>$.

35. Çelen Ş, Eğlence-Bakır S, Şahin M, Deniz I, Celik H, Kizilcikli I. Synthesis and characterization of new thiosemicarbazonato molybdenum(VI) complexes and their in vitro antimicrobial activities. Journal of Coordination Chemistry. 2019 May 19;72(10):1747-58. $\leq \mathrm{DOI}$.

36. Plyler EK. Infrared spectra of methanol, ethanol, and n-propanol. J Res Natl Bur Stand. 1952;48(4):281-6.

37. Vrdoljak V, Cindrić M, Milić D, MatkovićČalogović D, Novak P, Kamenar B. Synthesis of five new molybdenum(VI) thiosemicarbazonato complexes. Crystal structures of salicylaldehyde and 3-methoxy-salicylaldehyde 4methylthiosemicarbazones and their molybdenum(VI) complexes. Polyhedron. 2005 Sep;24(13):1717-26. <DOI>.

38. Novak P, Pičuljan K, Hrenar T, Smrečki V. Structure and Hydrogen Bonding Interactions in Methoxysalicylaldehyde Thiosemicarbazone Derivatives in Solution by NMR and DFT Methods. Croatica Chemica Acta. 2009;82(2):477-83. 


\section{Dioxomolybdenum(VI) Complexes of ONN-Chelating} Thiosemicarbazones: Crystallographic and Spectroscopic (UV, IR, and NMR) Studies

\section{Songül Eğlence-Bakır ${ }^{\mathrm{a} *}$}

aDepartment of Chemistry, Faculty of Science, Istanbul University, 34134, Istanbul, Turkey

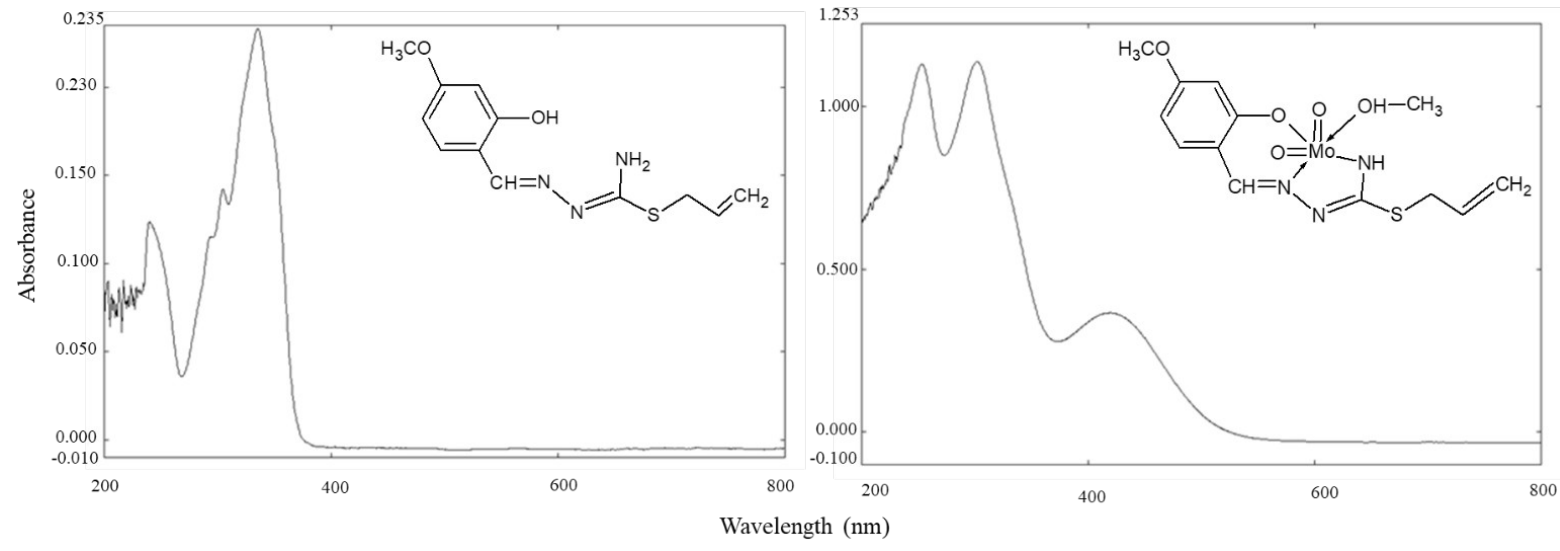

Figure S1: UV spectra of $\mathbf{L}^{\mathbf{1}}$ and $\mathbf{1}$
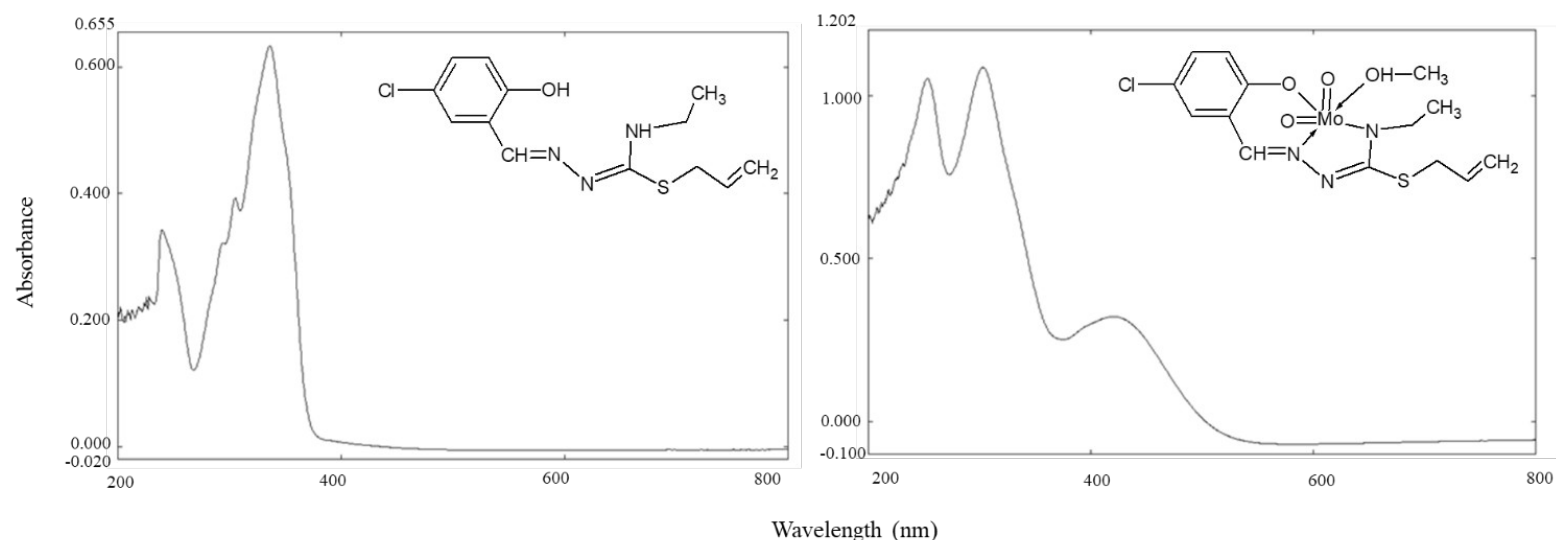

Figure S2: UV spectra of $\mathbf{L}^{2}$ and $\mathbf{2}$ 


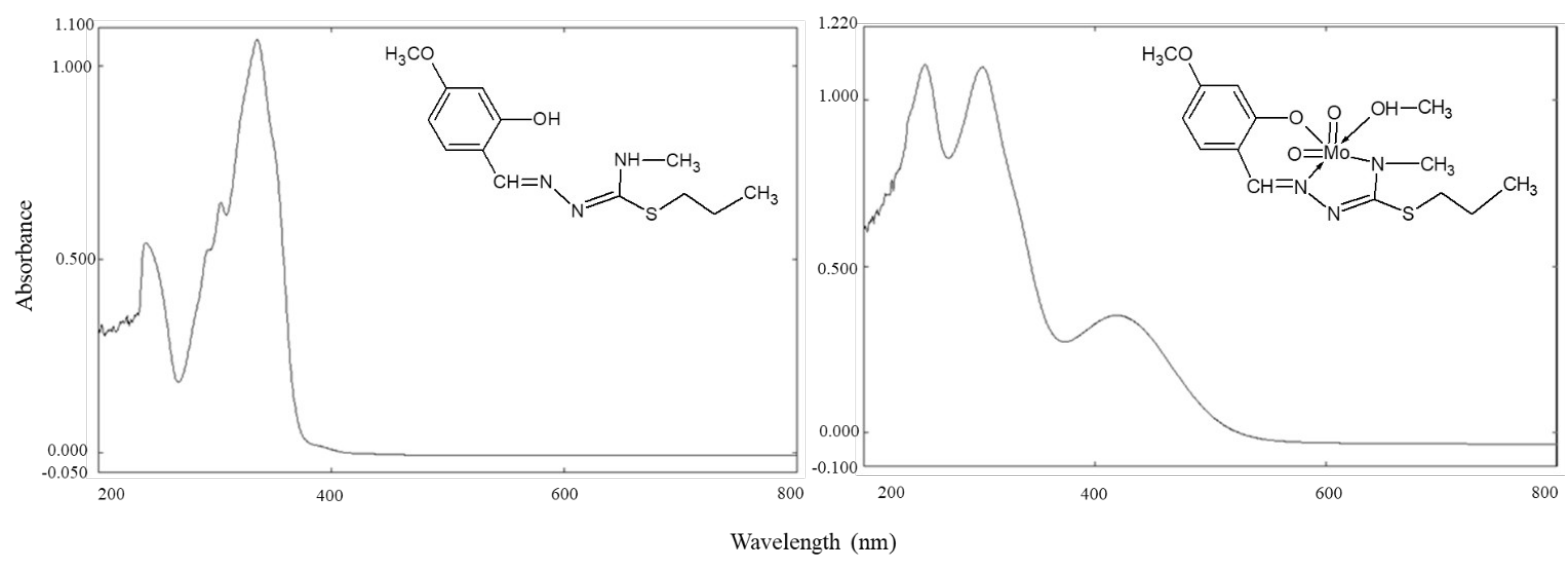

Figure S3: UV spectra of $\mathbf{L}^{\mathbf{3}}$ and $\mathbf{3}$

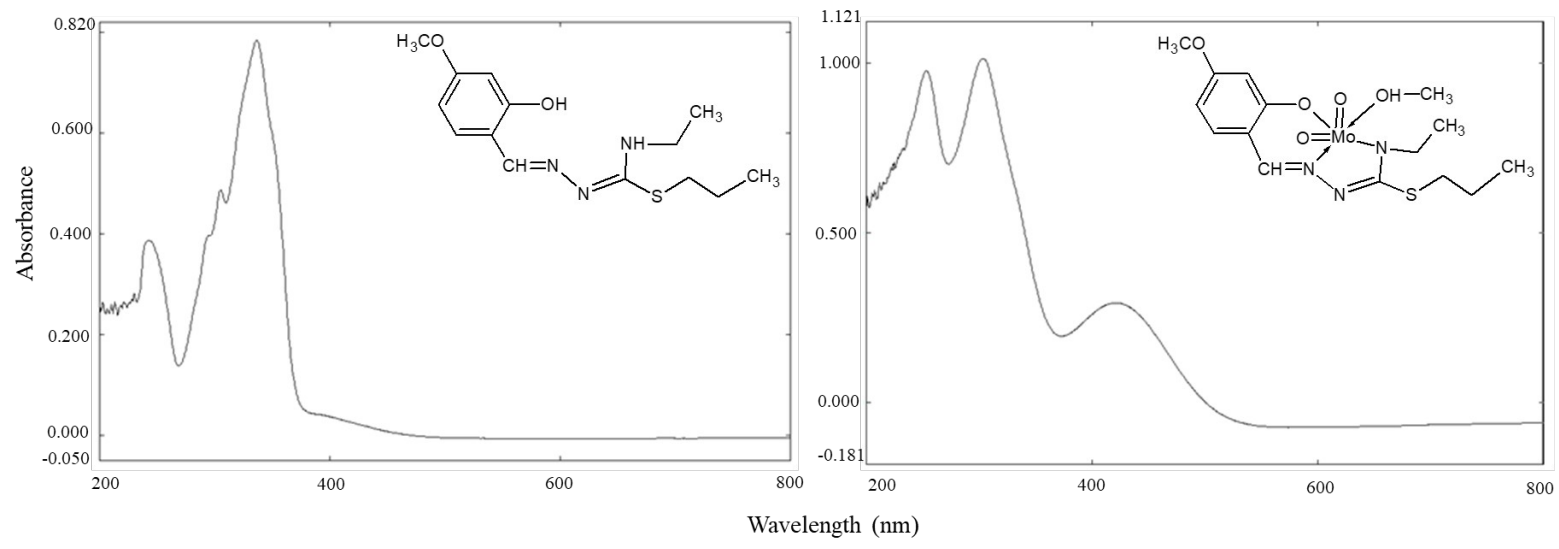

Figure S4: UV spectra of $L^{4}$ and $\mathbf{4}$ 


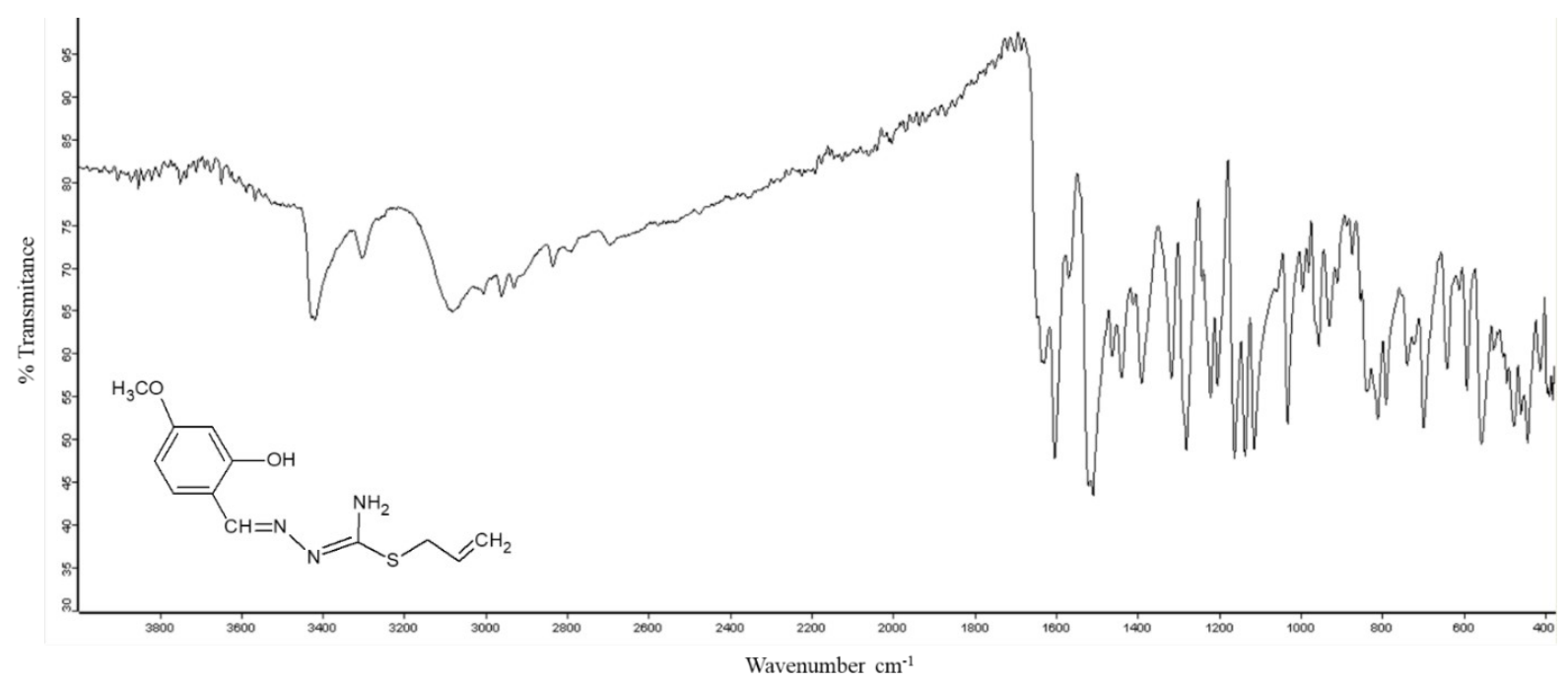

Figure S5: IR spectrum of $\mathbf{L}^{\mathbf{1}}$

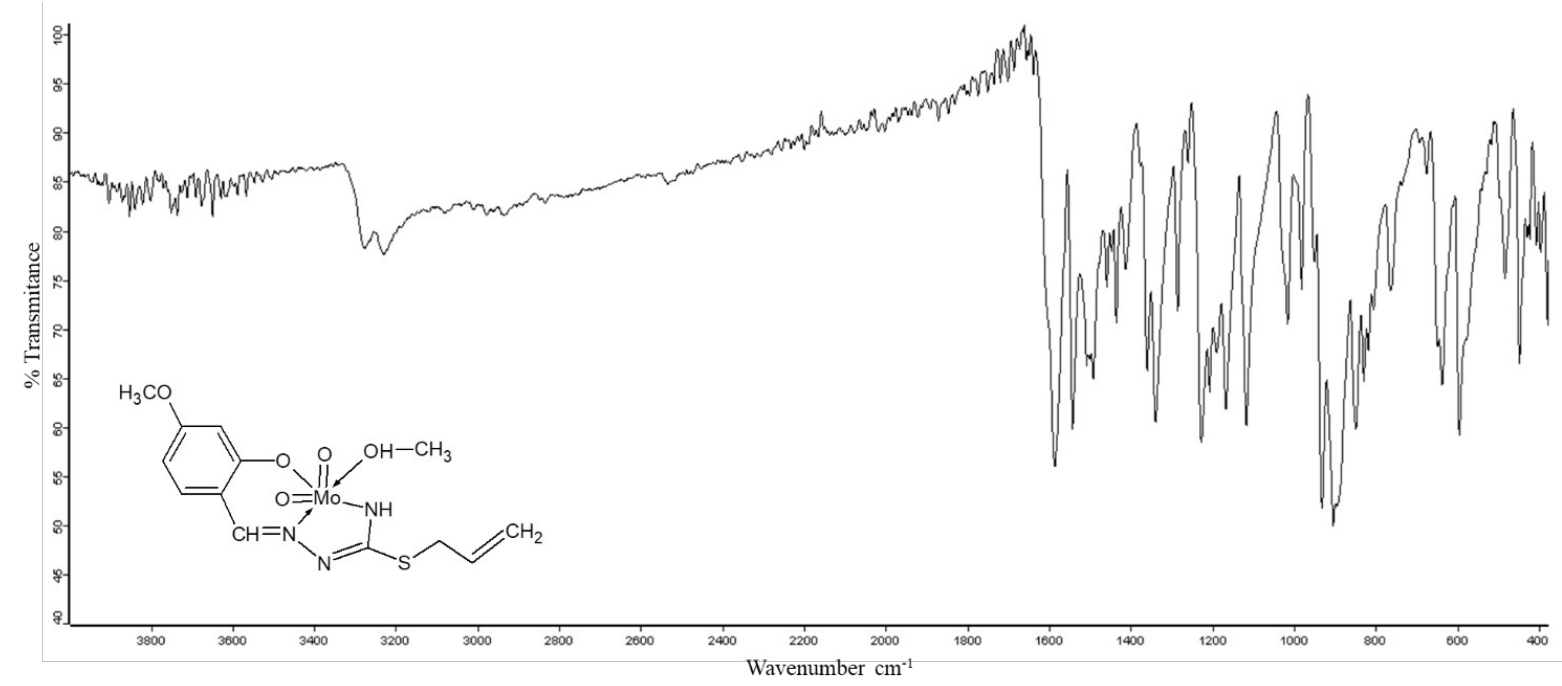

Figure S6: IR spectrum of 1 


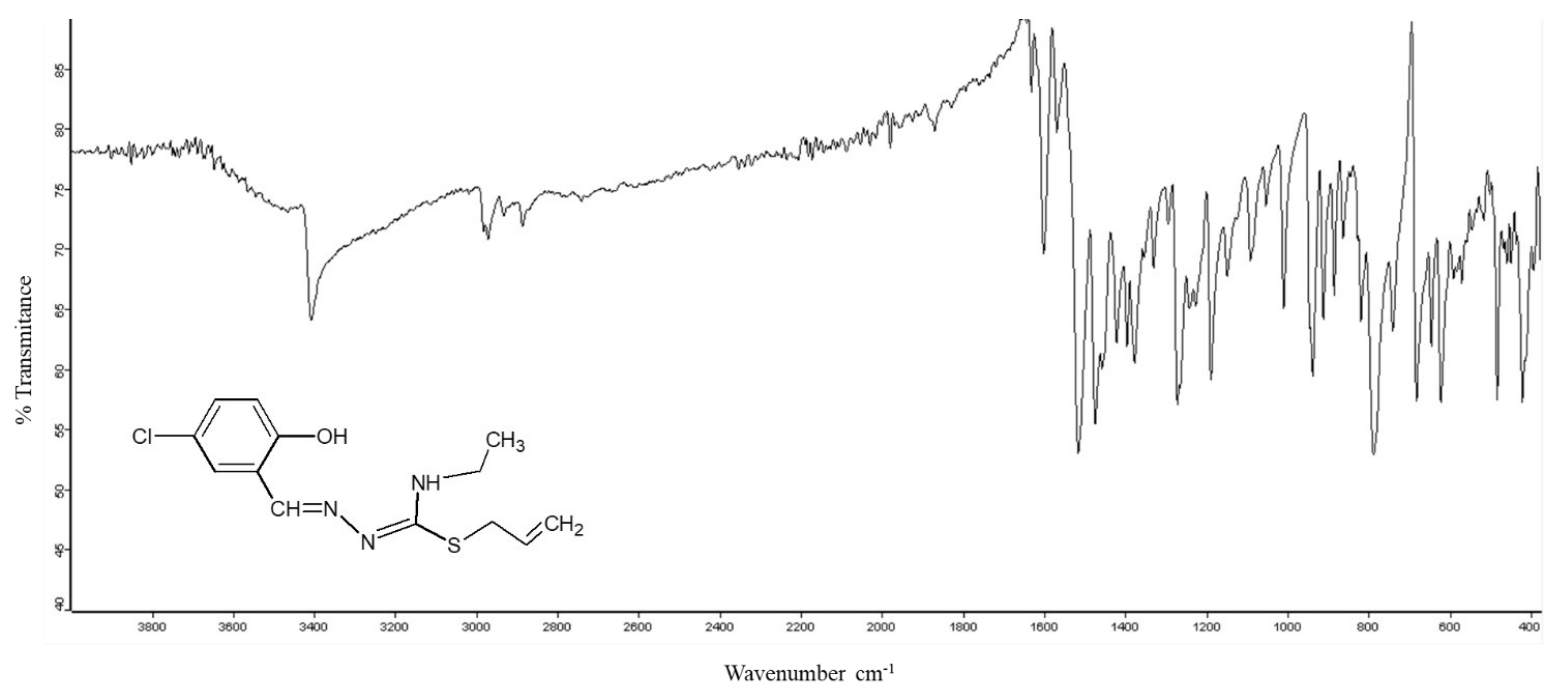

Figure S7: IR spectrum of $\mathbf{L}^{2}$

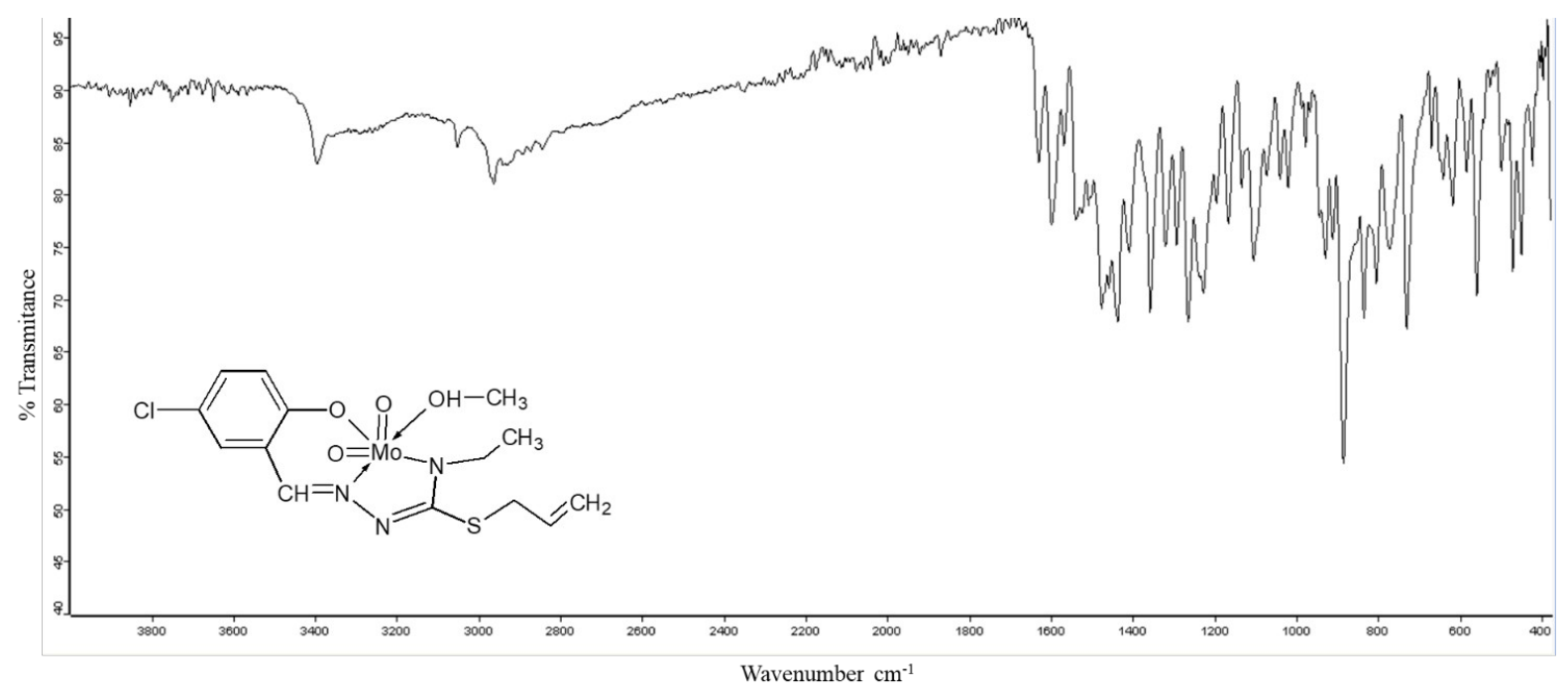

Figure S8: IR spectrum of $\mathbf{2}$ 


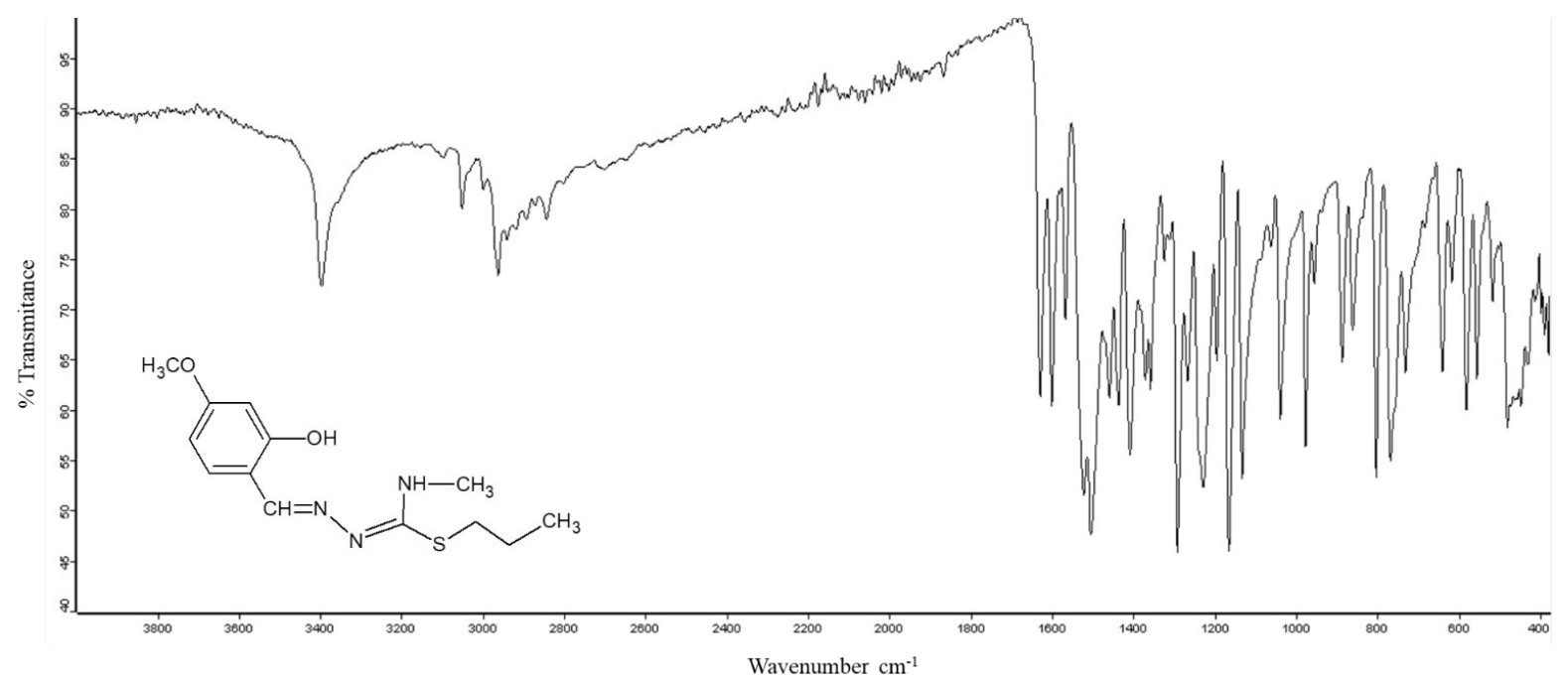

Figure S9: IR spectrum of $\mathbf{L}^{3}$

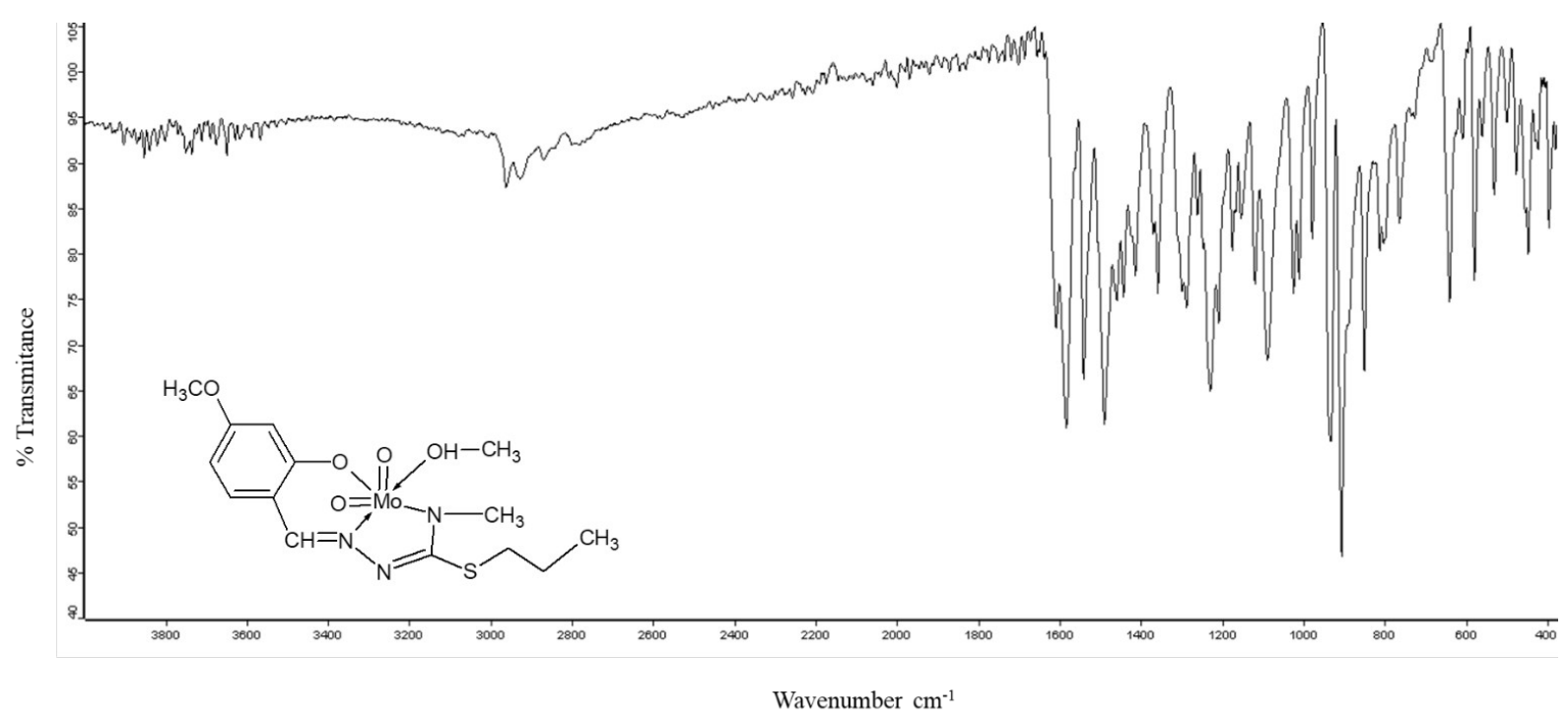

Figure S10: IR spectrum of $\mathbf{3}$ 


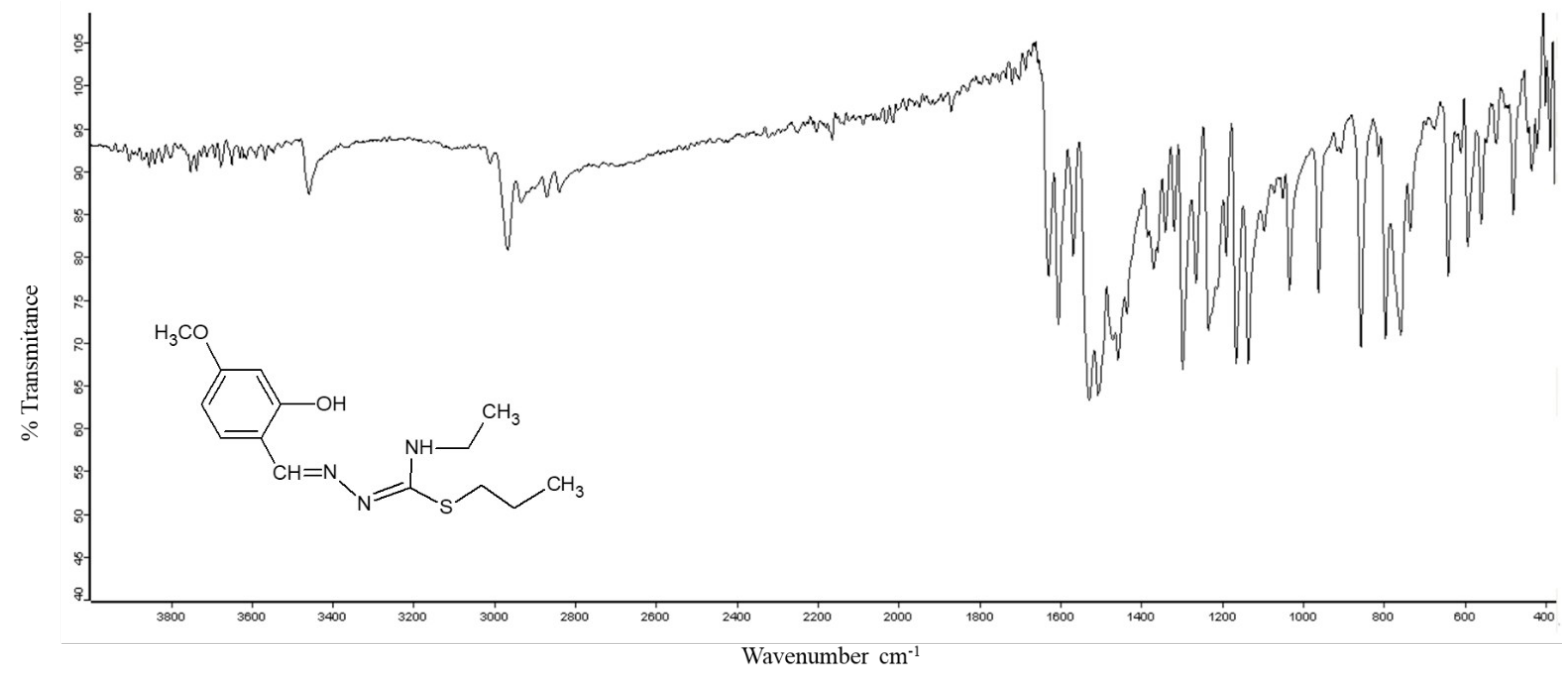

Figure S11: IR spectrum of $\mathbf{L}^{4}$

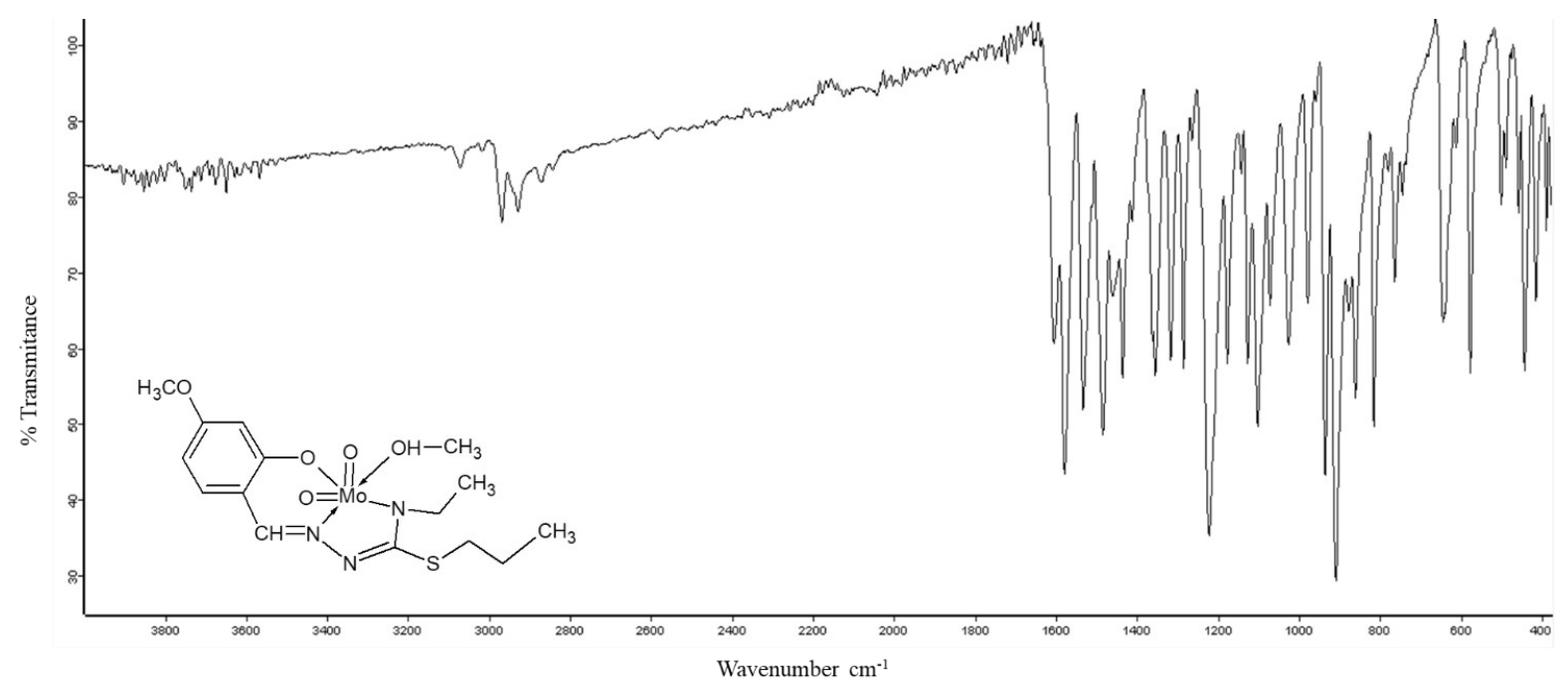

Figure S12: IR spectrum of 4 
Eğlence-Bakır S. JOTCSA. 2021; 8(4): 1275-1300.

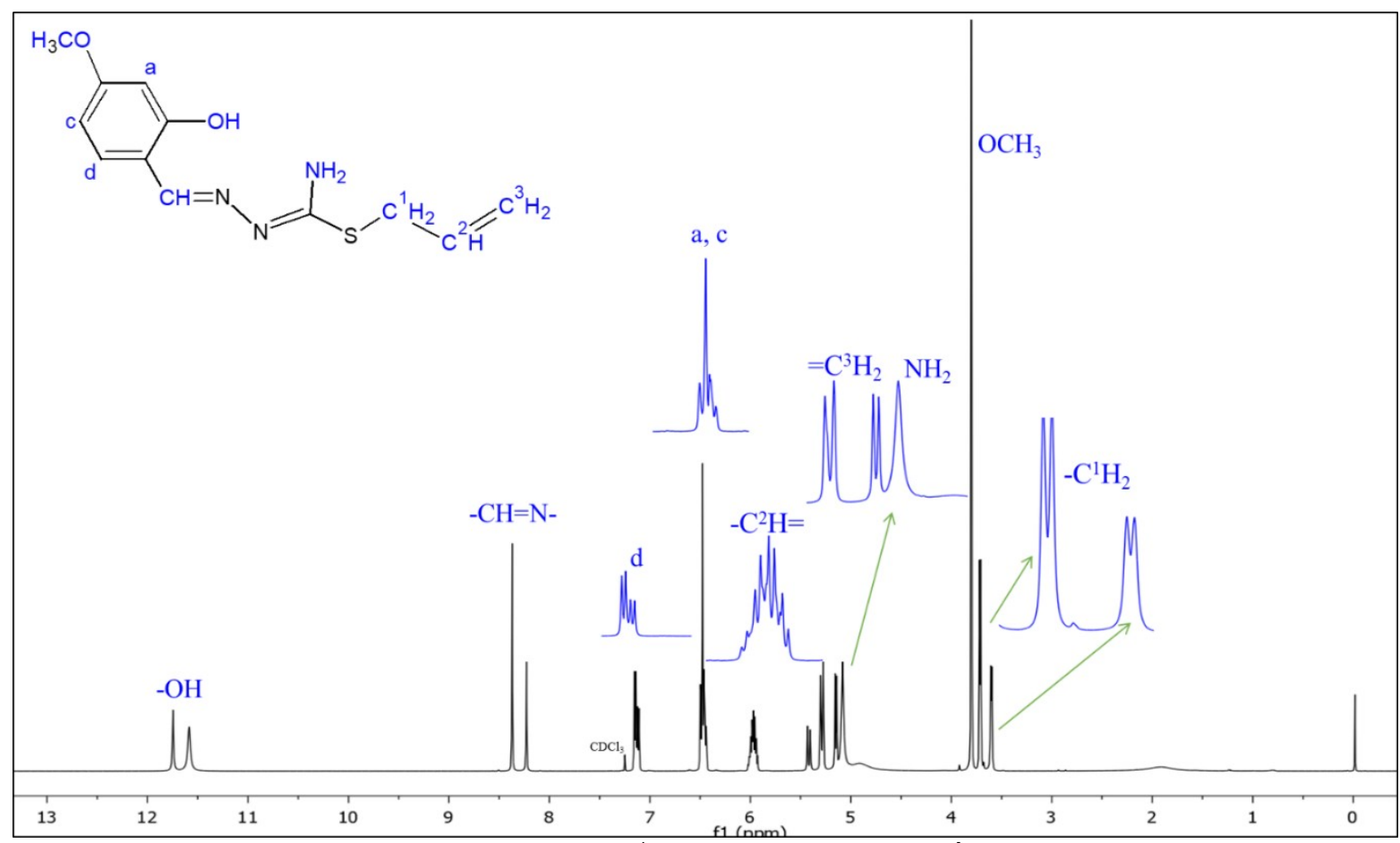

Figure S13: ${ }^{1} \mathrm{H}-\mathrm{NMR}$ spectrum of $\mathbf{L}^{\mathbf{1}}$

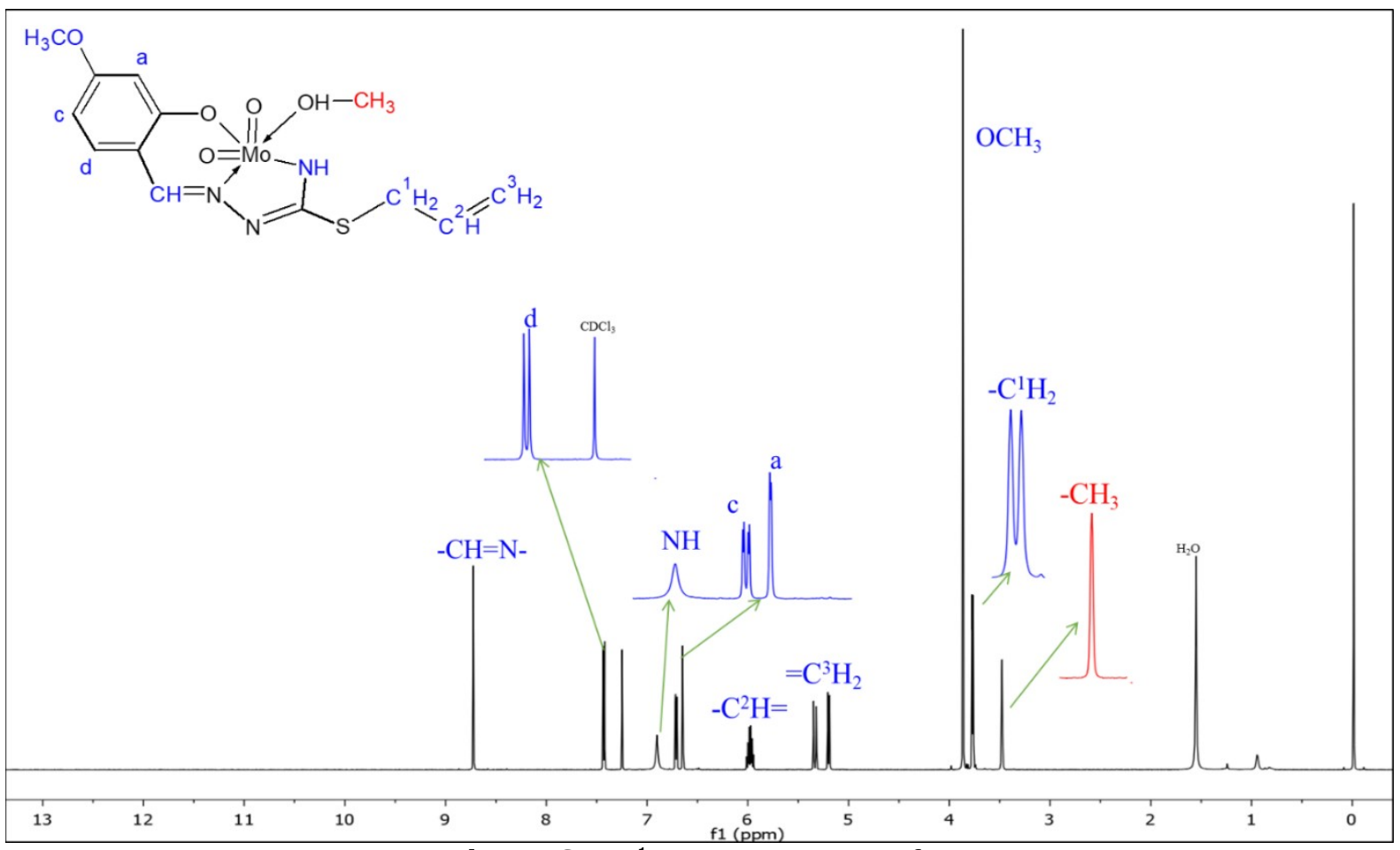

Figure S14: ${ }^{1} \mathrm{H}-\mathrm{NMR}$ spectrum of $\mathbf{1}$ 


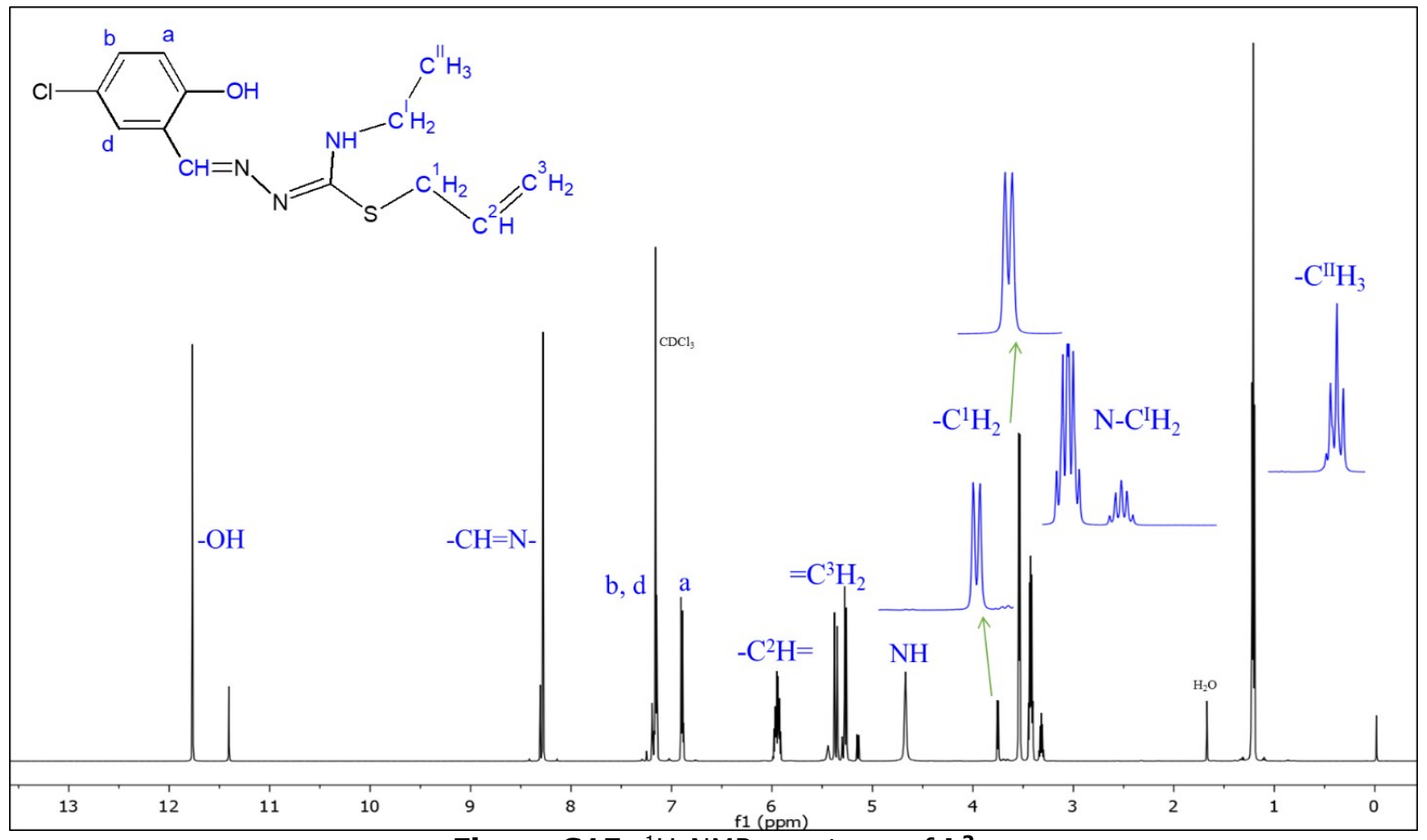

Figure S15: ${ }^{1} \mathrm{H}-\mathrm{NMR}$ spectrum of $\mathbf{L}^{\mathbf{2}}$

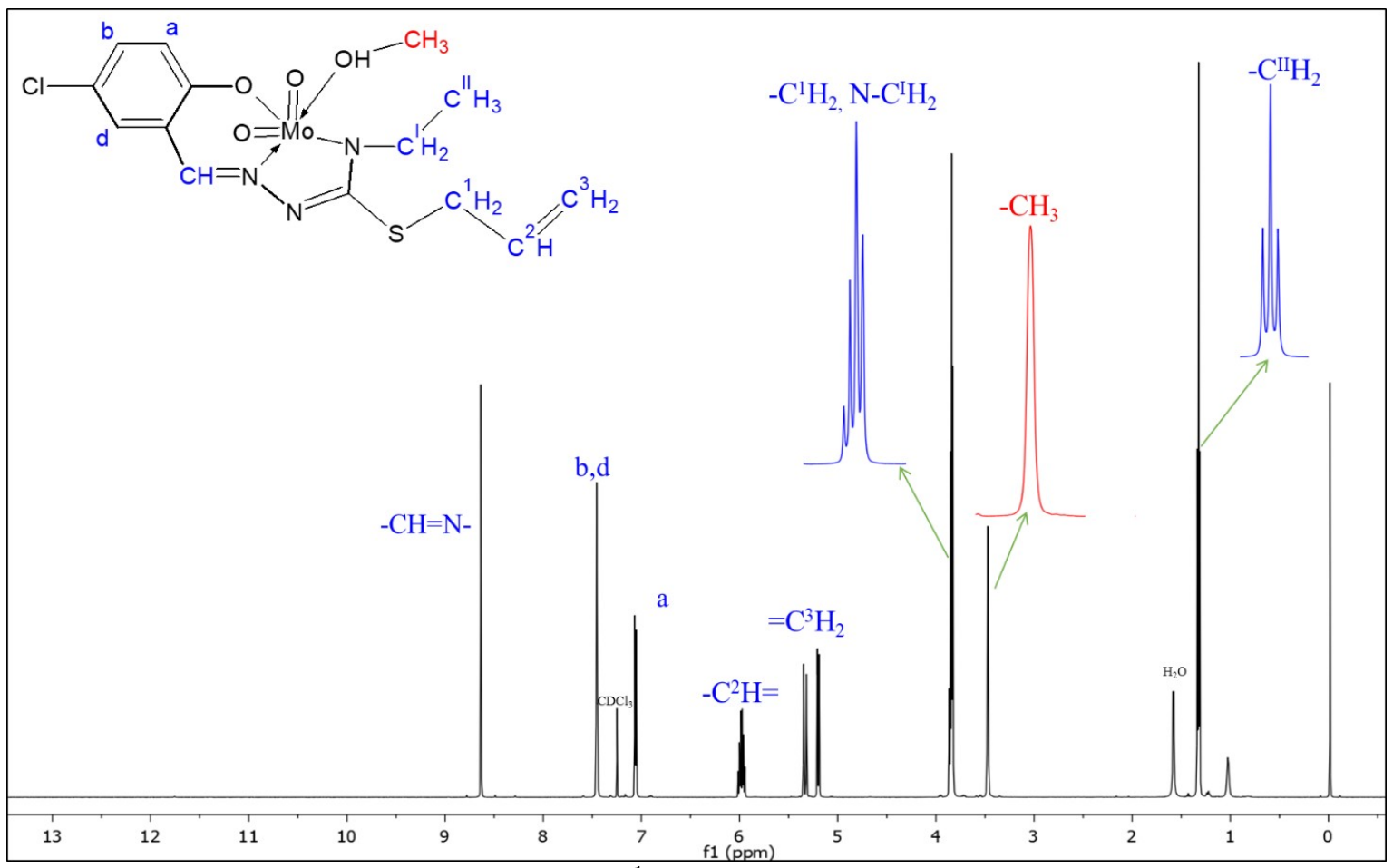

Figure S16: ${ }^{1} \mathrm{H}-\mathrm{NMR}$ spectrum of $\mathbf{2}$ 


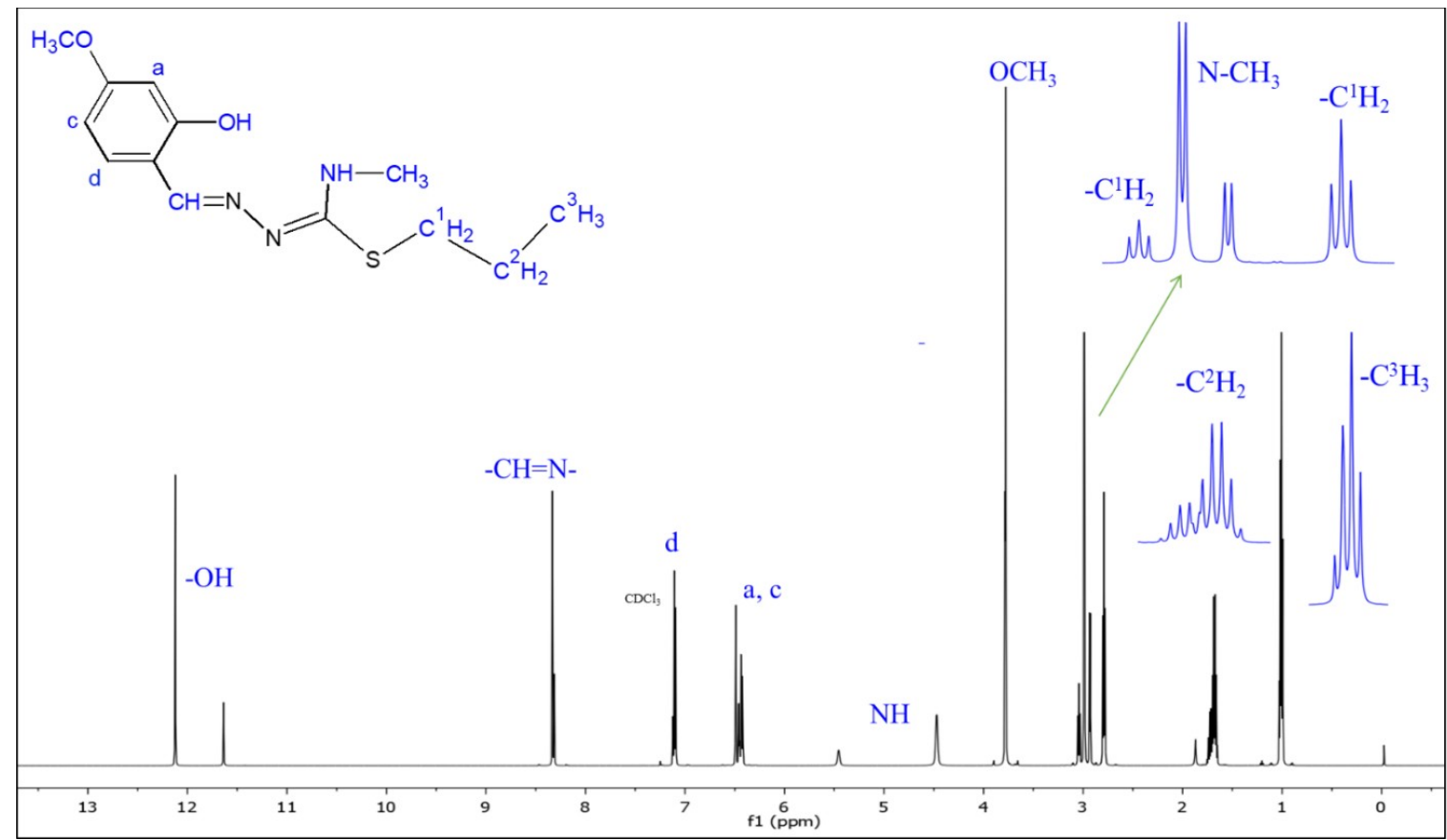

Figure S17: ${ }^{1} \mathrm{H}-\mathrm{NMR}$ spectrum of $\mathbf{L}^{\mathbf{3}}$

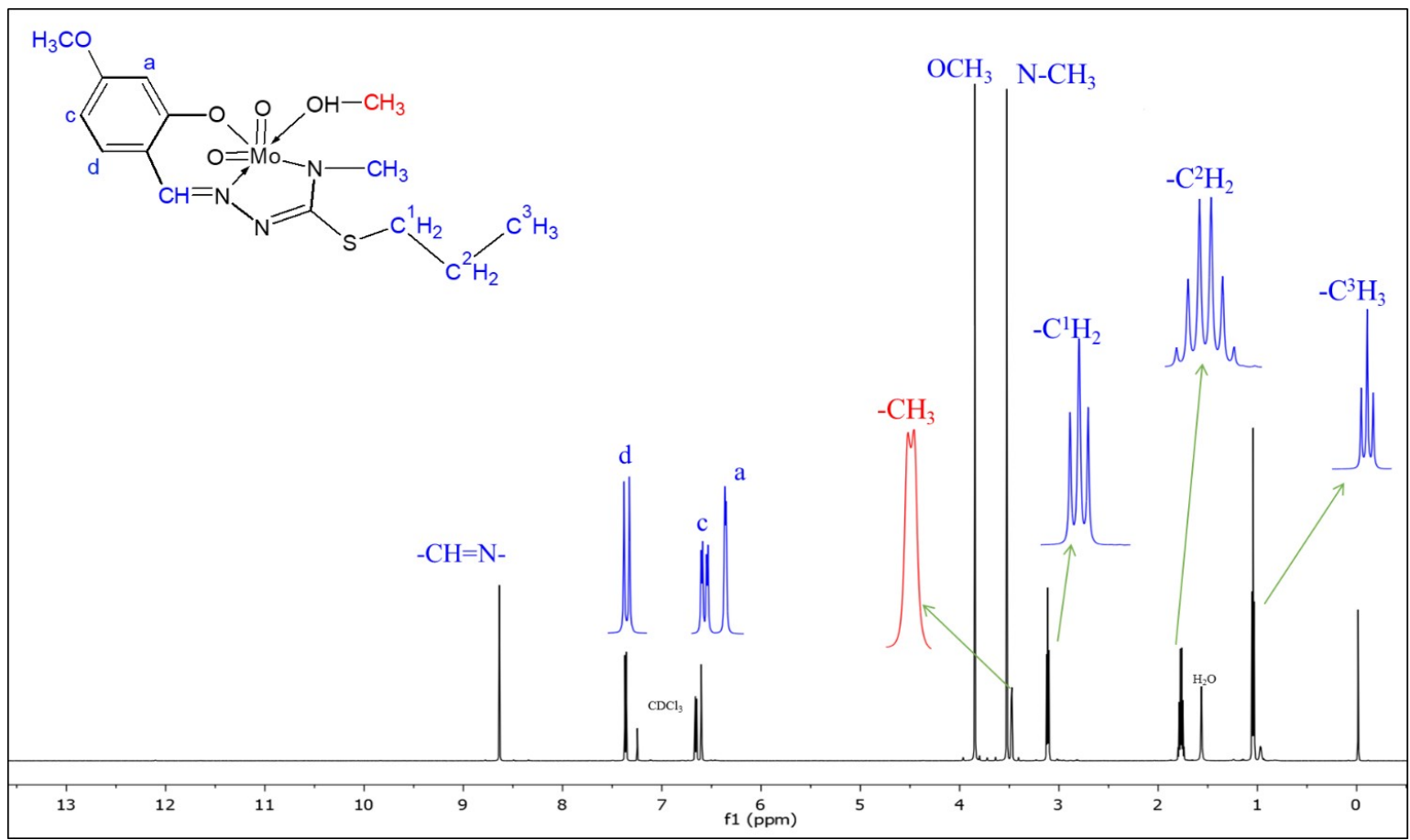

Figure S18: ${ }^{1} \mathrm{H}-\mathrm{NMR}$ spectrum of $\mathbf{3}$ 


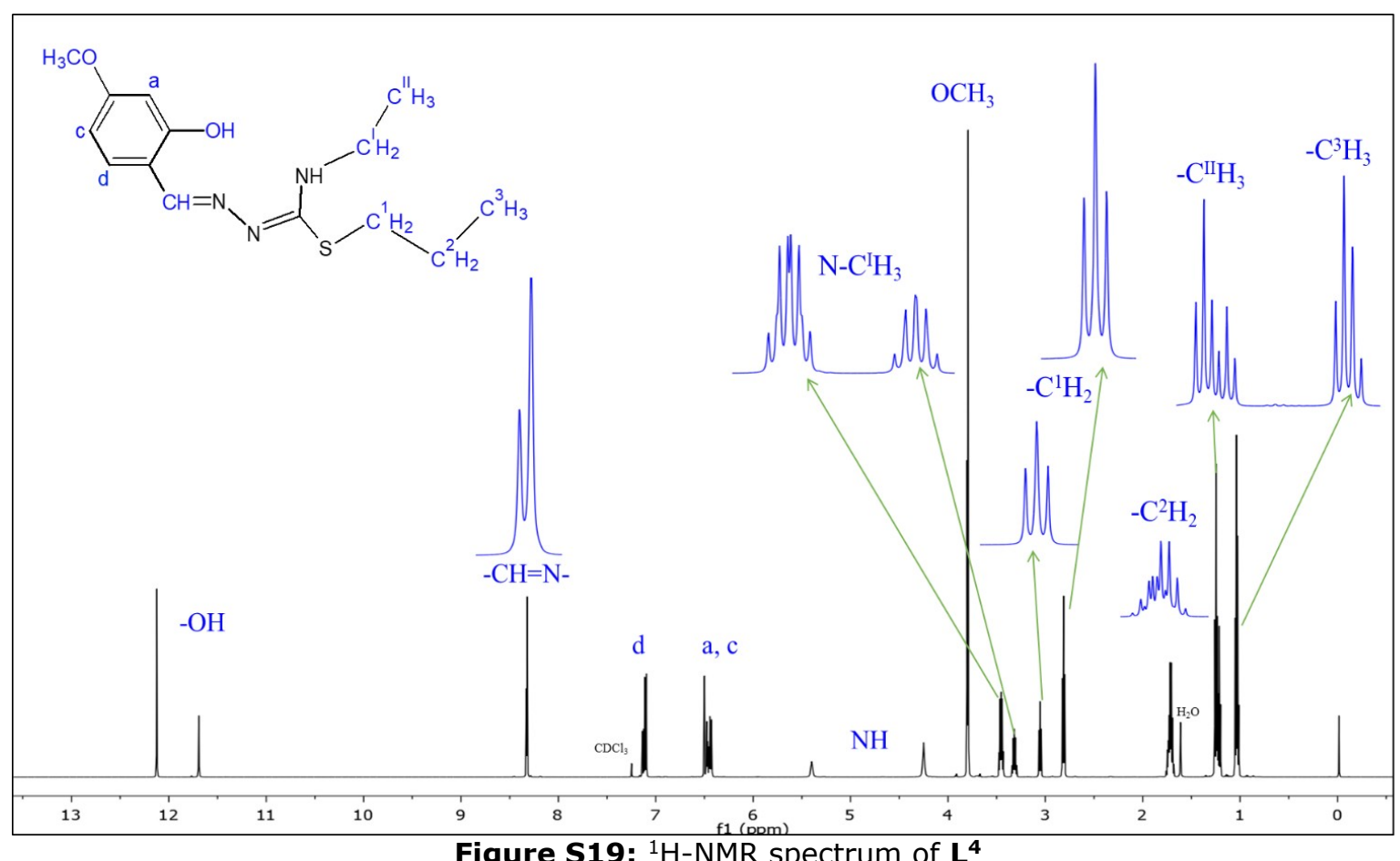

Figure S19: ${ }^{1} \mathrm{H}-\mathrm{NMR}$ spectrum of $\mathbf{L}^{4}$

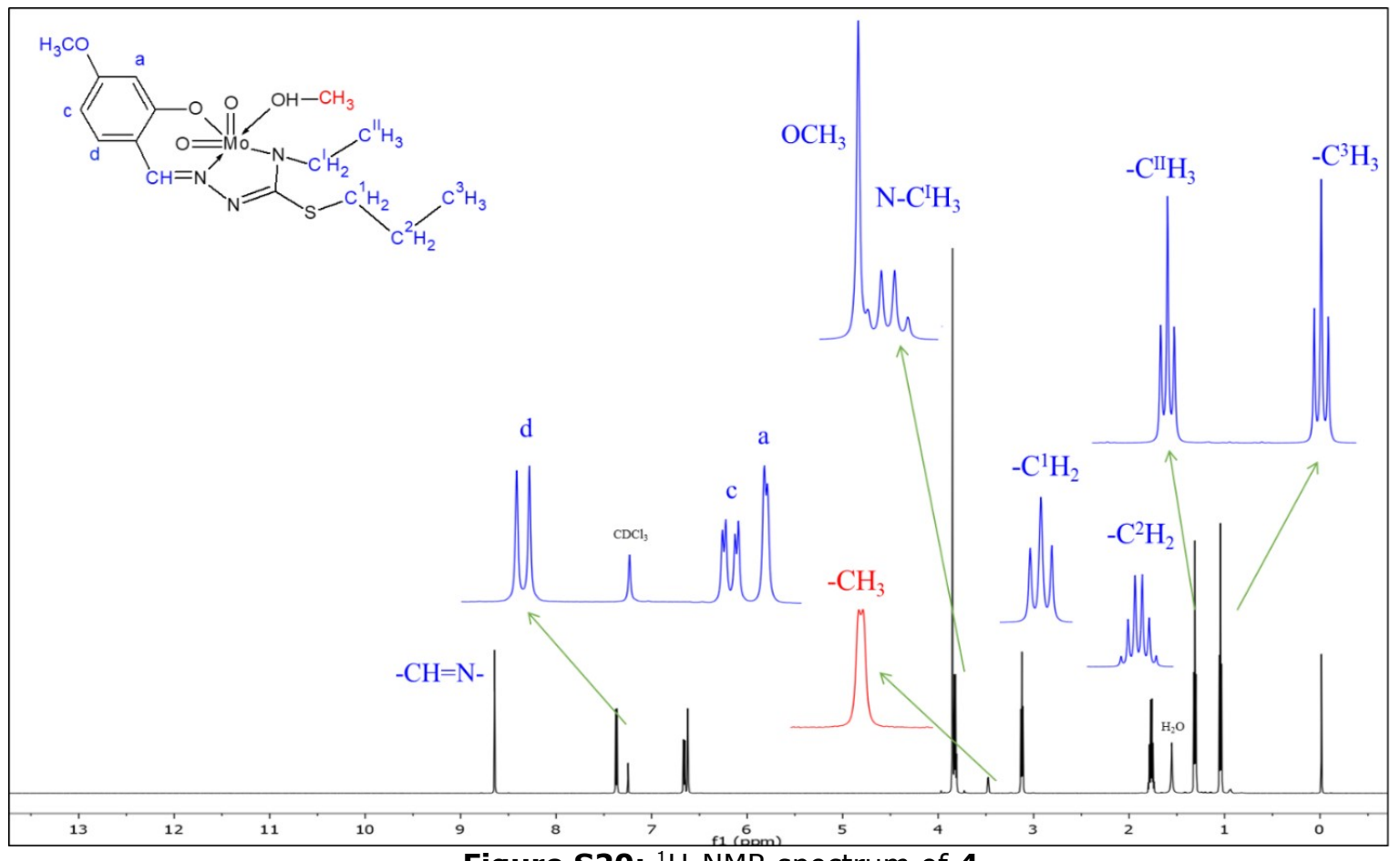

Figure S20: ${ }^{1} \mathrm{H}-\mathrm{NMR}$ spectrum of 4 


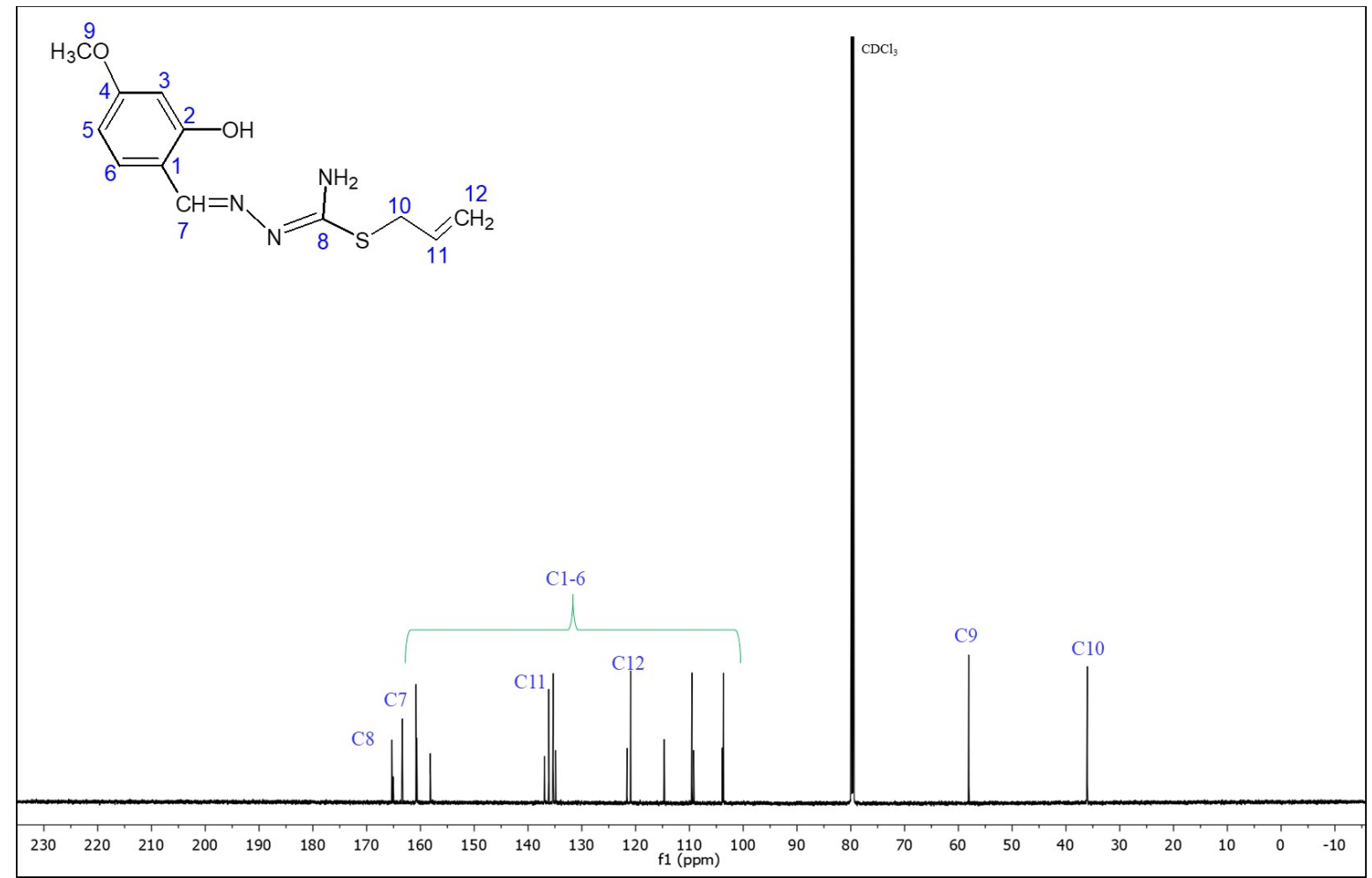

Figure S21: ${ }^{13} \mathrm{C}-\mathrm{NMR}$ spectrum of $\mathbf{L}^{\mathbf{1}}$

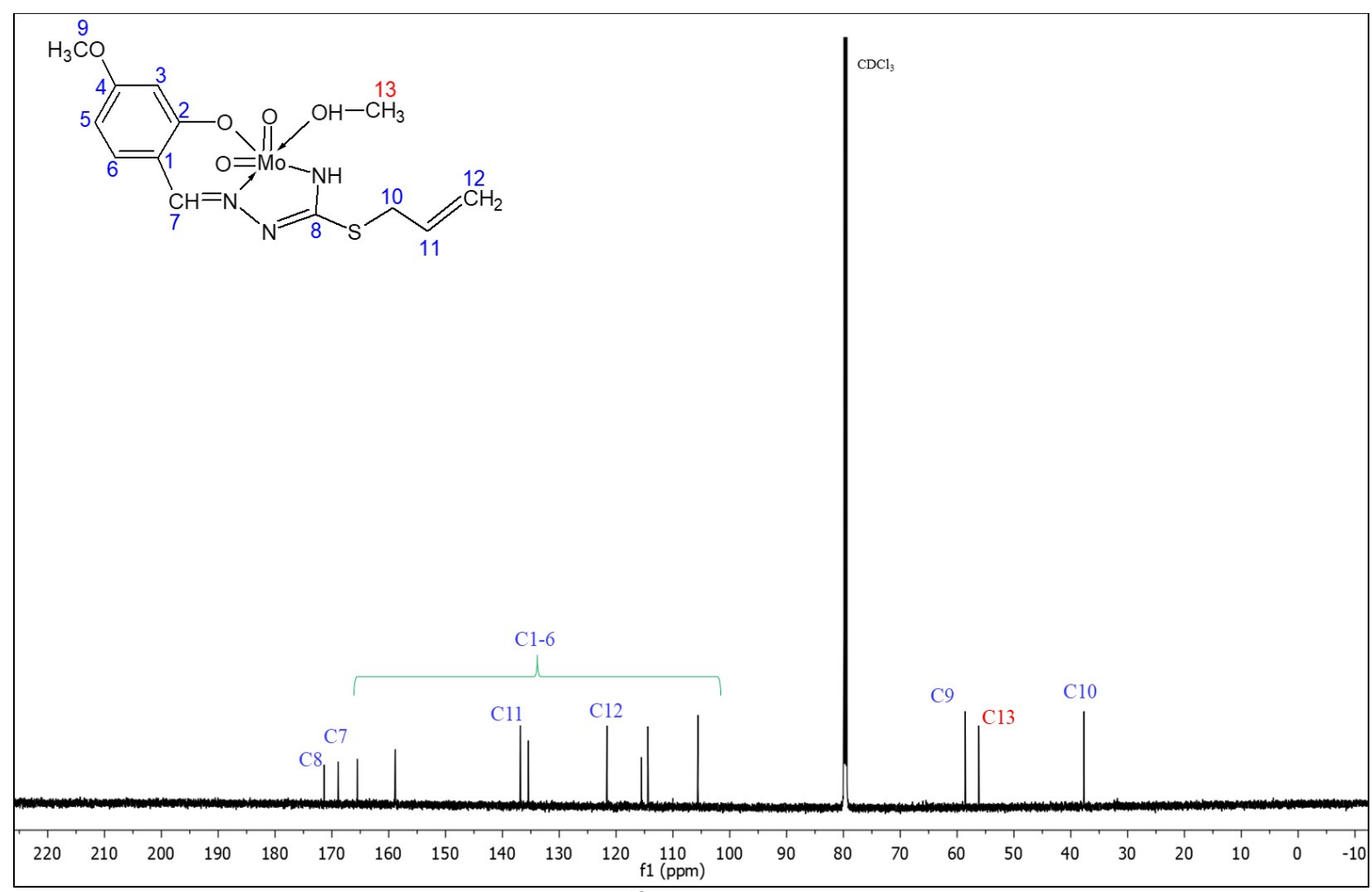

Figure S22: ${ }^{13} \mathrm{C}-\mathrm{NMR}$ spectrum of $\mathbf{1}$ 


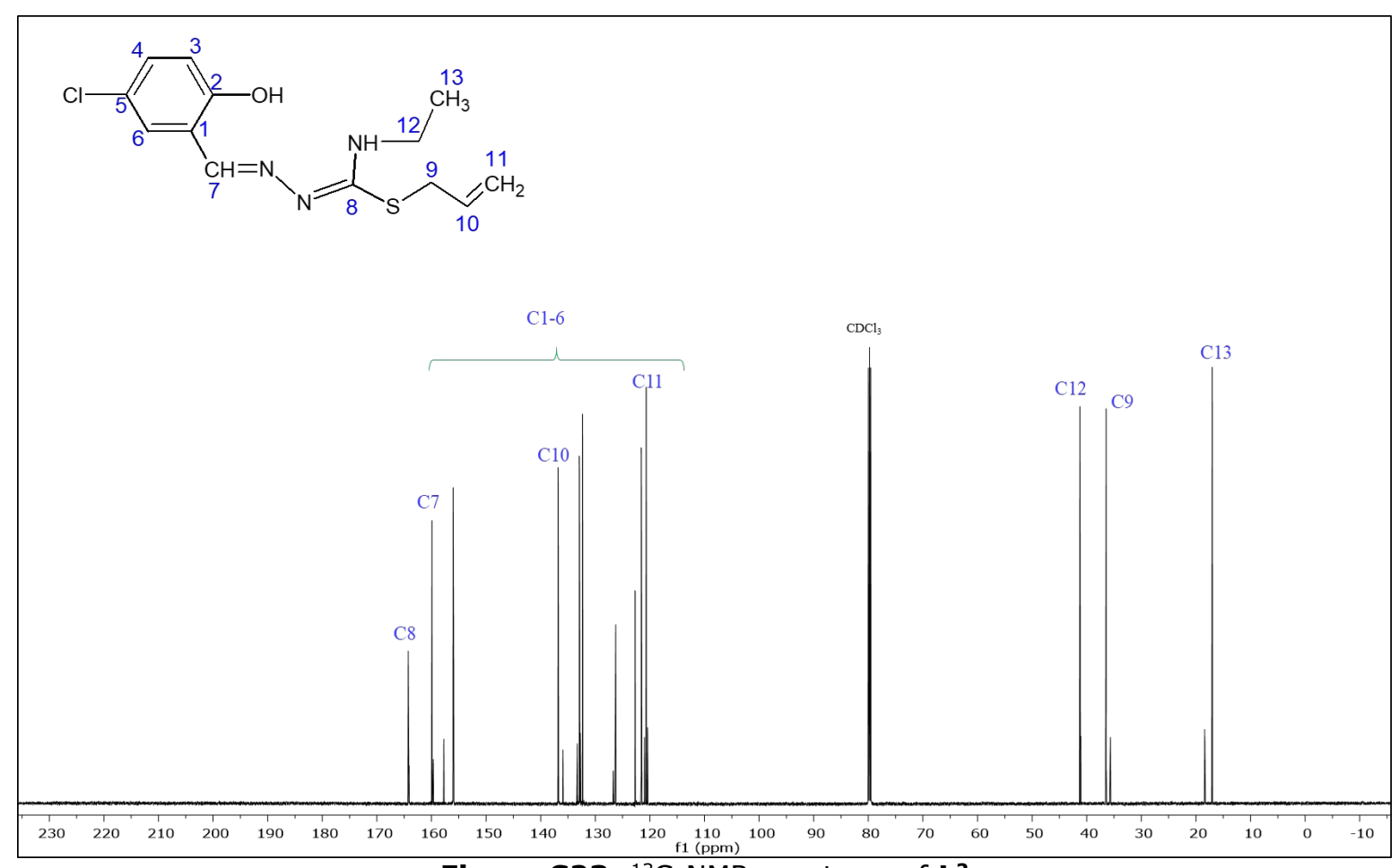

Figure S23: ${ }^{13} \mathrm{C}-\mathrm{NMR}$ spectrum of $\mathbf{L}^{2}$

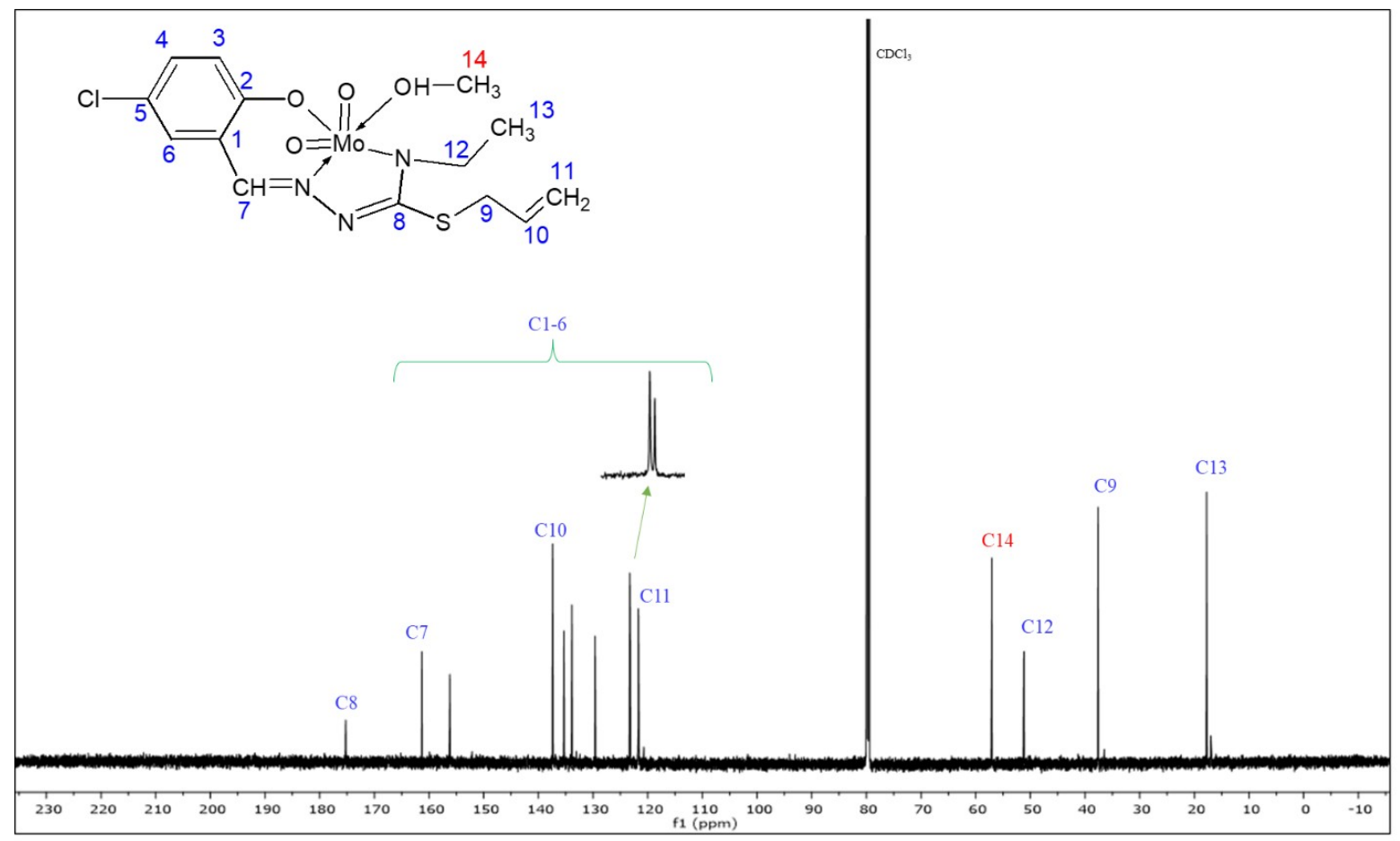

Figure S24: ${ }^{13} \mathrm{C}-\mathrm{NMR}$ spectrum of $\mathbf{2}$ 


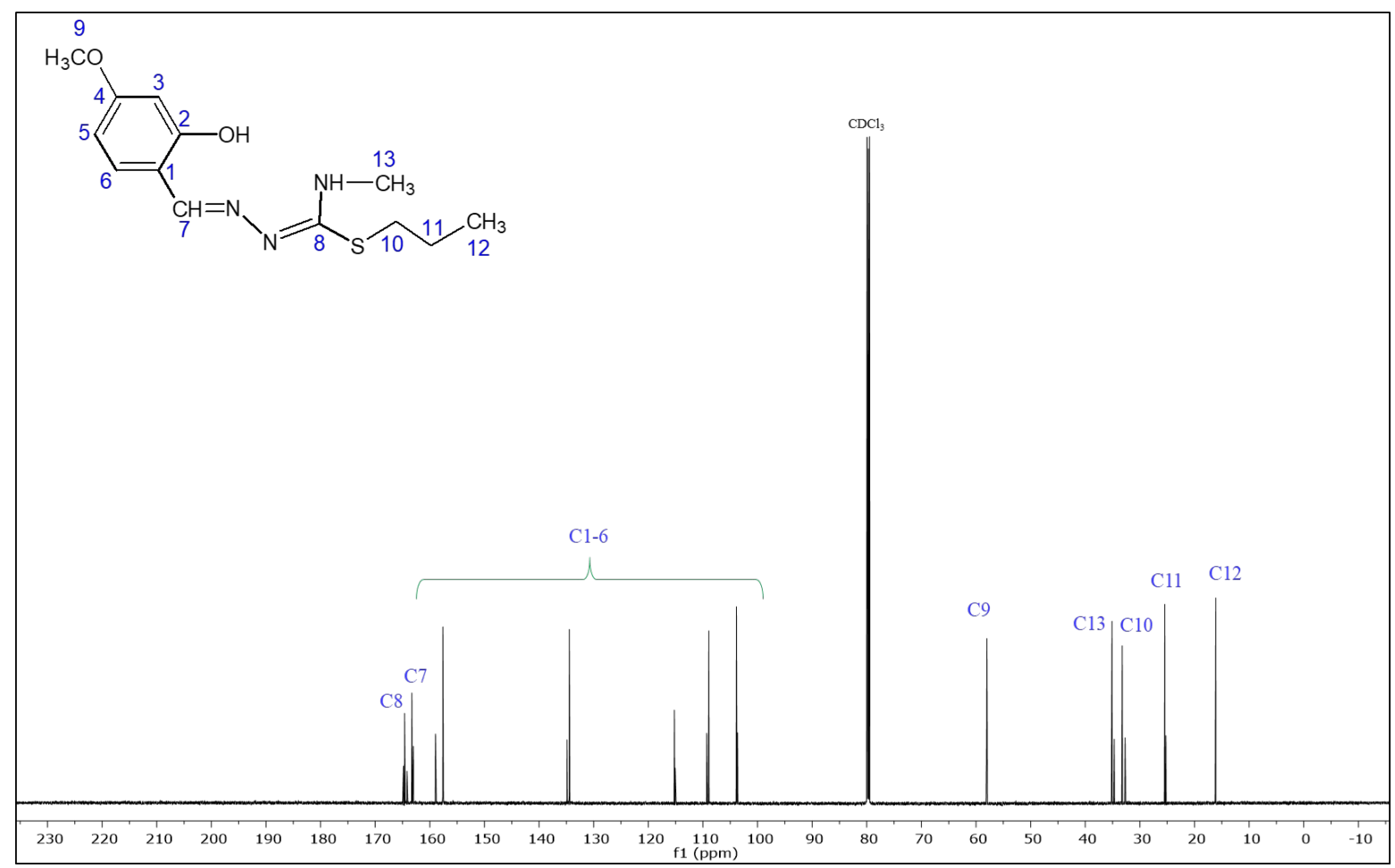

Figure S25: ${ }^{13} \mathrm{C}-\mathrm{NMR}$ spectrum of $\mathbf{L}^{\mathbf{3}}$

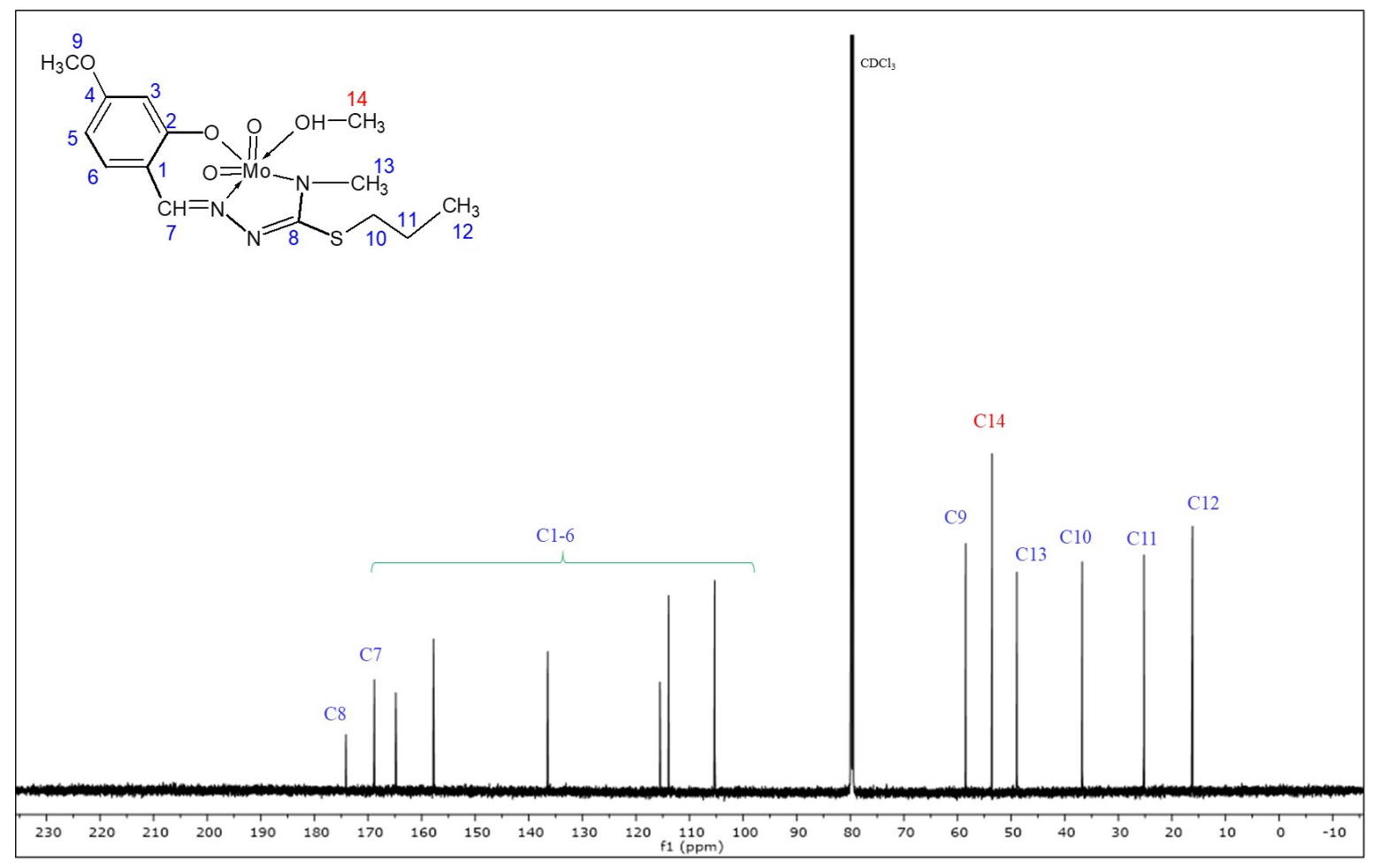

Figure S26: ${ }^{13} \mathrm{C}-\mathrm{NMR}$ spectrum of $\mathbf{3}$ 


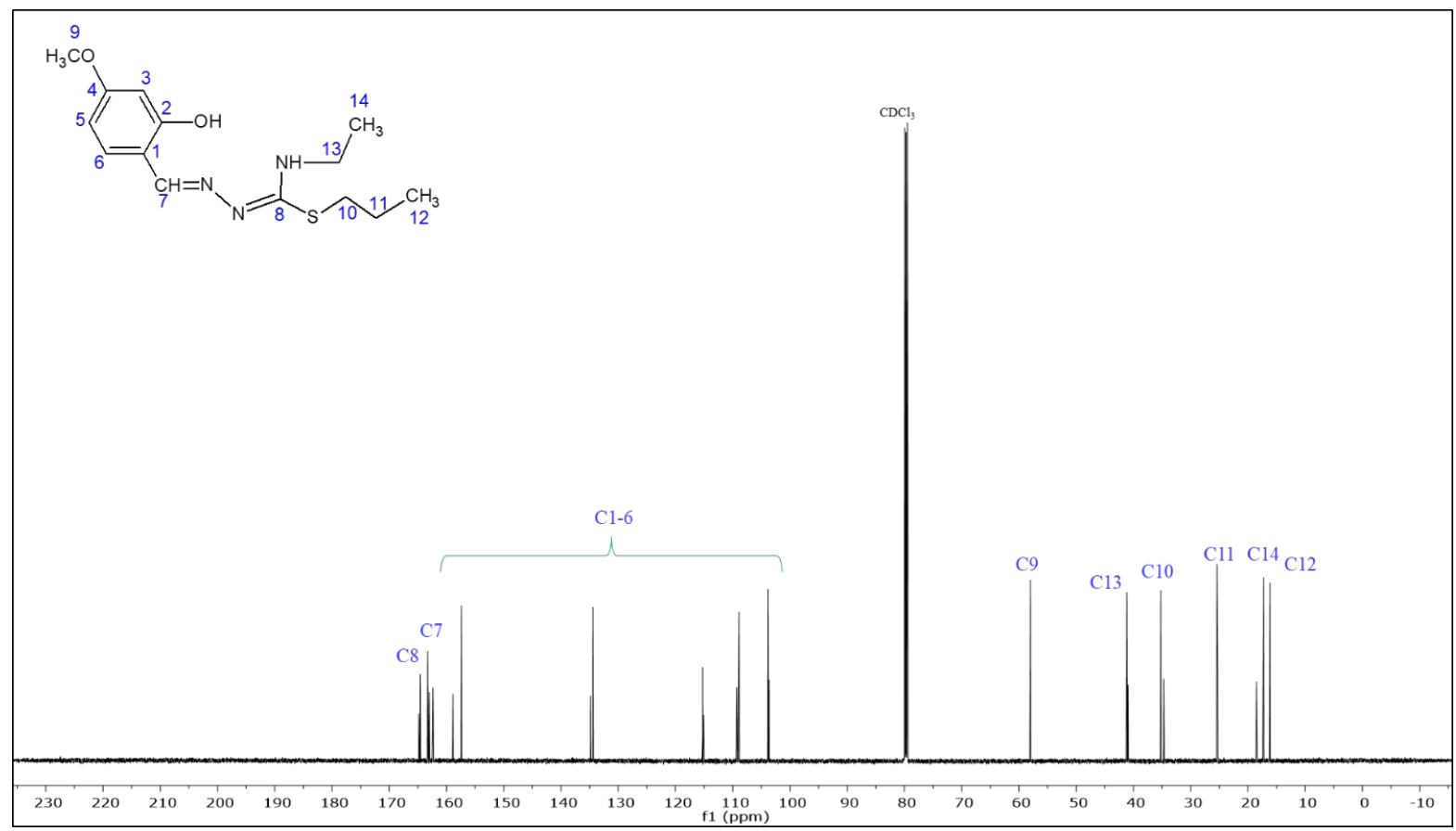

Figure S27: ${ }^{13} \mathrm{C}$-NMR spectrum of $\mathbf{L}^{4}$

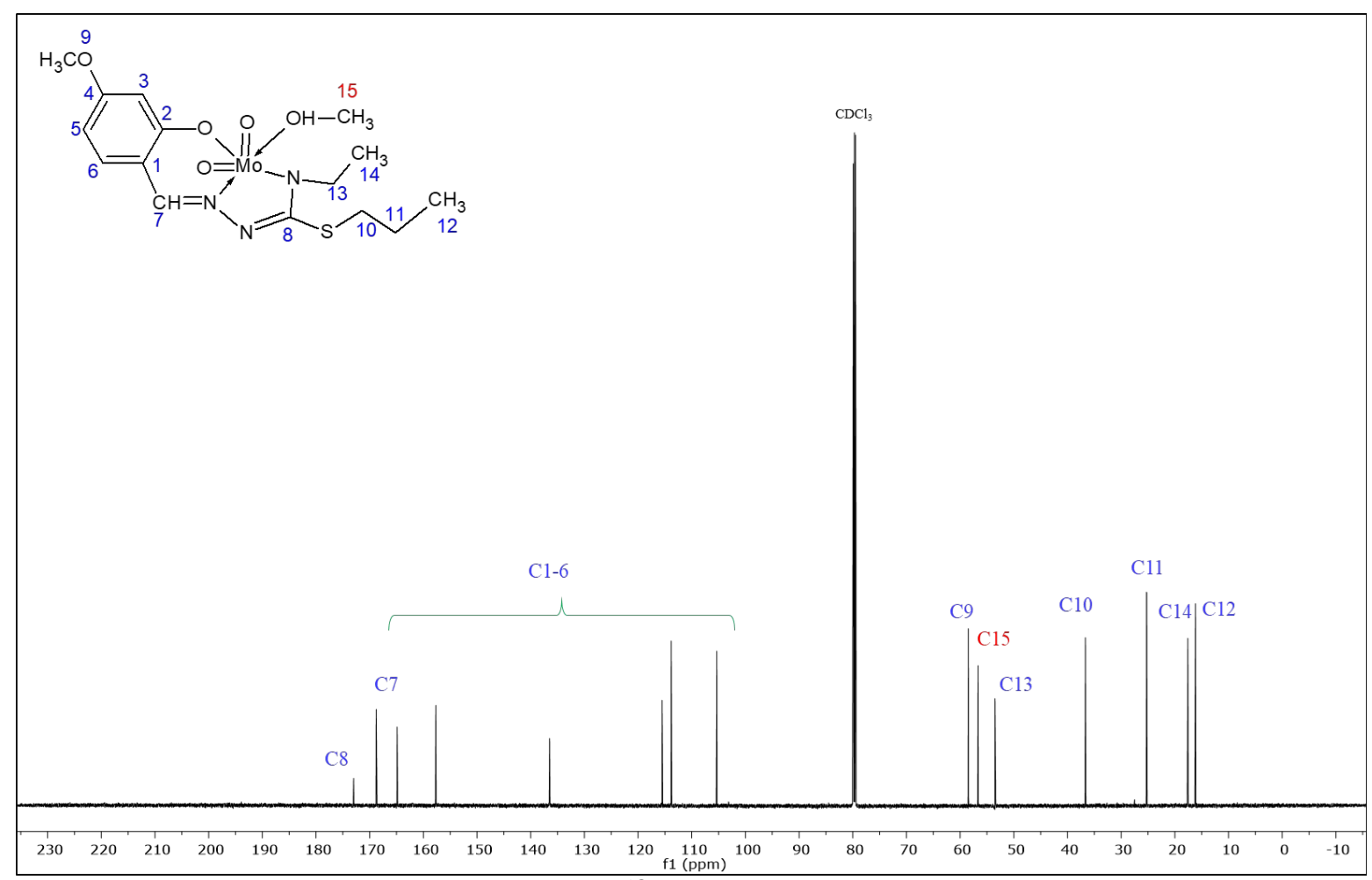

Figure S28: ${ }^{13} \mathrm{C}-\mathrm{NMR}$ spectrum of 4 